\title{
Global Analysis of Fragmentation Functions for Pions and Kaons and Their Uncertainties
}

\author{
Daniel de Floriar* and Rodolfo Sassot \\ Departamento de Fisica, Universidad de Buenos Aires, \\ Ciudad Universitaria, Pab. 1 (1428) Buenos Aires, Argentina \\ Marco Stratmann \\ Radiation Laboratory, RIKEN, 2-1 Hirosawa, Wako, Saitama 351-0198, Japan
}

\begin{abstract}
We present new sets of pion and kaon fragmentation functions obtained in NLO combined analyses of single-inclusive hadron production in electron-positron annihilation, proton-proton collisions, and deep-inelastic lepton-proton scattering with either pions or kaons identified in the final state. At variance with all previous fits, the present analyses take into account data where hadrons of different electrical charge are identified, which allow to discriminate quark from anti-quark fragmentation functions without the need of non trivial flavor symmetry assumptions. The resulting sets are in good agreement with all data analyzed, which cover a much wider kinematical range than in previous fits. An extensive use of the Lagrange multiplier technique is made in order to assess the uncertainties in the extraction of the fragmentation functions and the synergy from the complementary data sets in our global analysis.
\end{abstract}

PACS numbers: 13.87.Fh, 13.85.Ni, 12.38.Bx

\section{INTRODUCTION AND MOTIVATION}

The hadronization process turns partons produced in hard-scattering reactions into the physical, colorless, non perturbative hadronic bound states towards any hard interaction ultimately develops. Within the standard framework of leading-twist collinear QCD [1], processes with an observed hadron in the final-state can be described in terms of perturbative hard scattering cross sections and certain non-perturbative but universal functions: parton distributions, accounting for the partonic structure of the hadrons in the initial state just before the interaction, and fragmentation functions, encoding the details of the subsequent hadronization process [2].

These three ingredients are therefore the pillars of the perturbative QCD (pQCD) description of hard interactions; their precise knowledge has been crucial for its success in the past, and is imperative for the ongoing and future high energy research programs [3]. In the last few years, the improvement in each of these key areas has been remarkable. Higher order QCD calculations have been explored and validated for most processes up to next-to-leading order (NLO) accuracy, and are currently being extended even beyond that point for some observables [4].

The knowledge on parton distributions has become increasingly precise as a result of two decades of a wide variety of high precision measurements, and strenuous efforts to update and enlarge periodically the corresponding QCD analyses [5, 6]. State-of-the-art sets of parton

\footnotetext{
*Electronic address: deflo@df.uba.ar

${ }^{\dagger}$ Electronic address: sassot@df.uba.ar

‡Electronic address: marco@ribf.riken.jp
}

densities agree with each other well within the already fairly small estimated uncertainties, and provide a picture of the proton structure fully consistent with the data. For most observables, the differences arising from the use of one or another modern set of parton densities are negligibly small compared with the uncertainties in their measurement or from unknown higher order corrections.

Also fragmentation functions have been rapidly evolving, following the path of parton densities, however, without attaining yet the precision of the latter [7, 8, 9, 10]. Most of the information used to determine these distributions comes essentially from electron-positron annihilation into charged hadrons. These data have the important advantages of being very precise, thanks to high statistics measurements from CERN-LEP and at SLAC [11, 12, 13, 14, 15, 16] and clean, in the sense that the corresponding cross sections have no dependence on parton densities.

In spite of these advantages, electron-positron annihilation data suffer from many shortcomings. In the first place, the data give per se no information on how to disentangle quark from anti-quark fragmentation as they always refer to the charge sum for a certain hadron species, e.g., $\pi^{+}+\pi^{-}$. The information on how the individual quark flavors fragment into hadrons depends crucially on so-called "tagging" techniques and the underlying assumptions implemented in the Monte-Carlo generators employed [14]. In addition to that, at the mass scale of the $Z$-boson, i.e., for the bulk of the electronpositron annihilation data, all electro-weak couplings become roughly equal and thus only flavor singlet combinations of fragmentation functions can be determined.

Also the gluon fragmentation is not exceedingly well constrained, since the sub-leading NLO corrections for electron-positron annihilation are too weak to determine 
it; quark-gluon mixing in the scale evolution of fragmentation functions is not enough of a constraint either, due to the lack of precise enough data at energy scales away from the $Z$-resonance. The data also become considerably less accurate and sparse at large hadron energy fractions, leading to large uncertainties in that region.

Fortunately, in the last few years several one-particle inclusive measurements coming from both proton-proton collisions and deep-inelastic lepton-nucleon scattering have matured enough as to yield complementary information on the fragmentation process with competing precision [18, 19, 20, 21, 22]. These measurements not only probe fragmentation in a complementary energy regime, but also weigh quite differently contributions of individual parton flavors in the hadronization process.

Including these data in the extraction of fragmentation functions, not only increases statistics but, most importantly, yields a much more complete picture of the fragmentation process. For example, the complementary information allows to relax and test certain rather stringent assumptions on the flavor symmetry of fragmentation functions usually made. One can also make an independent check of the flavor tagging techniques implemented in electron-positron annihilation, and, ultimately, scrutinize the fundamental ideas of QCD factorization and the universality of the fragmentation functions.

In this paper, we perform for the first time such a more comprehensive global QCD analysis to obtain new sets of pion and kaon fragmentation functions in agreement with the wealth of electron-positron annihilation data and also electron-proton and proton-proton observables. The latter were either impossible to calculate with previous sets or just not reproduced by them. As it should be expected, our new sets agree with previous extractions [7, 8, 9, 10] in aspects of fragmentation functions which are actually determined well by electron-positron data but show significant differences otherwise.

In order to asses the uncertainties in the resulting fragmentation functions, associated with both the uncertainties in the data and the theoretical estimate of the observables, we have made an extensive use of the Lagrange multiplier method 23]. We study in detail the profiles of the $\chi^{2}$-function that quantifies the agreement between the set of fragmentation functions, more specifically their moments, and the data. This procedure gives a clear picture of the relative uncertainty for each quark flavor and the gluon, which are found to be about $3 \%$ and $10 \%$ for "favored" (valence) fragmentation functions in pions and kaons, respectively, and of the order of $10 \%$ and $20 \%$ for the respective "unfavored" (sea) fragmentation functions. The method allows also to assess the role and interplay of the various data sets in constraining the different fragmentation functions, illustrating the synergy characteristic of a global analysis.

In the following Section, we briefly summarize the QCD framework for fragmentation functions and the different single-inclusive hadron production processes included in the global analysis. In Sec. III, we outline the details of the analysis, discussing our choice for the functional form used to parametrize the fragmentation functions at the initial scale of the scale evolution, and the data sets included in the fit. We also outline the implementation of the Mellin transform method for a fast evaluation of the NLO cross sections, and the Lagrange multiplier technique for assessing uncertainties. In Sec. IV we discuss in great detail our results for both pion and for kaon fragmentation functions and their uncertainties. We briefly summarize our results in Sec. V.

\section{QCD FRAMEWORK FOR FRAGMENTATION FUNCTIONS AND SINGLE-INCLUSIVE HADRON PRODUCTION}

The pQCD framework for single-inclusive hadron production in electron-positron annihilation, lepton-nucleon deep-inelastic scattering, and hadron-hadron collisions is in place for quite some time now, and calculations at NLO accuracy are "state-of-the-art" throughout. In each case one exploits the factorization theorem [1], which states that the cross section can be decomposed into appropriate convolutions of perturbatively calculable partonic hard scattering cross sections and certain combinations of non-perturbative parton distribution and fragmentation functions. We can restrict ourselves to a brief summary of theoretical framework relevant for our global QCD analysis of fragmentation functions to set up our notation in which we closely follow Refs. 24] and [25].

\section{A. Properties of Fragmentation Functions}

A field theoretical definition of fragmentation functions $D_{f}^{H}$ in terms of bi-local operators was given in Ref. [2] and reads for quarks (up to kinematical pre-factors)

$$
\begin{aligned}
D_{q}^{H}(z) \propto & \int d x^{-} e^{-i P_{H}^{+} x^{-} / z} \\
& \operatorname{Tr}\left[\gamma^{+}\left\langle 0|\Psi(0) \mathcal{P}| H\left(P_{H}^{+}\right) X\right\rangle\left\langle H\left(P_{H}^{+}\right) X\left|\mathcal{P}^{\prime} \bar{\Psi}(x)\right| 0\right\rangle\right]
\end{aligned}
$$

and similarly for gluons. $\mathcal{P}$ and $\mathcal{P}^{\prime}$ denote the necessary gauge-links to render (1) gauge-invariant. The simple parton model interpretation of $D_{f}^{H}(z)$ as the probability for a parton $f$ to produce a hadron $H$ with fraction $z$ of its momentum is recovered in light-cone gauge where $\mathcal{P}=\mathcal{P}^{\prime}=1$. Since a specific hadron $H$ with light-cone momentum $P_{H}^{+}$is observed in the final-state, a local operator product expansion (OPE) does not apply. For fully inclusive parton densities, the OPE is the basis for first principle computations of some of their integer moments within "lattice QCD". Similar calculations cannot be pursued for fragmentation functions.

The scale dependence of the fragmentation functions $D_{f}^{H}$ is calculable in pQCD and governed by renormalization group equations very similar to those for parton 
densities. For instance, the singlet evolution equation schematically reads

$$
\frac{d}{d \ln Q^{2}} \vec{D}^{H}\left(z, Q^{2}\right)=\left[\hat{P}^{(T)} \otimes \vec{D}^{H}\right]\left(z, Q^{2}\right),
$$

where

$$
\vec{D}^{H} \equiv\left(\begin{array}{c}
D_{\Sigma}^{H} \\
D_{g}^{H}
\end{array}\right), D_{\Sigma}^{H} \equiv \sum_{q}\left(D_{q}^{H}+D_{\bar{q}}^{H}\right)
$$

and

$$
\hat{P}^{(T)} \equiv\left(\begin{array}{cc}
P_{q q}^{(T)} & 2 n_{f} P_{g q}^{(T)} \\
\frac{1}{2 n_{f}} P_{q g}^{(T)} & P_{g g}^{(T)}
\end{array}\right) .
$$

is the matrix of the singlet timelike evolution kernels. The NLO splitting functions $P_{i j}^{(T)}$ have been computed in [26, 27] or can be related to the corresponding spacelike kernels by proper analytic continuation [28].

The range of applicability for fragmentation functions as defined above is severely limited to medium-to-large values of $z$. On the one hand, the timelike evolution kernels in (4) develop a strong singular behavior as $z \rightarrow 0$, and, on the other hand, the produced hadrons are considered to be massless. More specifically, the splitting functions $P_{g q}^{(T)}(z)$ and $P_{g g}^{(T)}(z)$ have a dominant, large logarithmic piece $\simeq \ln ^{2} z / z$ in their NLO part, which ultimately leads to negative fragmentation functions for $z \ll 1$ in the course of the $Q^{2}$ evolution and, perhaps, to unphysical, negative cross sections, even if the evolution starts with positive distributions at some scale $Q_{0}<Q$. At small $z$, also finite mass corrections proportional to $M_{H} /\left(s z^{2}\right)$ become more and more important. While there are ways to resum the singular small- $z$ behavior to all orders in $\alpha_{s}$, there is no systematic or unique way to correct for finite hadron masses, for instance by introducing some "re-scaled" variable $z^{\prime}$ in SIA. Inseparably entwined with mass effects are other power corrections or "dynamical higher twists".

Anyway, including small- $z$ resummations or mass corrections in one way or the other in the analysis of hadron production rates is not compatible with the factorization theorem and the definition of fragmentation functions outlined above. "Resummed" or "mass corrected" fragmentation functions should not be used with fixed order expressions for, say, the semi-inclusive deep-inelastic production of a hadron, $e N \rightarrow e^{\prime} H X$, discussed in Sec. IIC. Therefore we limit ourselves in our global analysis to kinematical regions where mass corrections and the influence of the singular small- $z$ behavior of the evolution kernels is negligible. It turns out that a cut $z>z_{\min }=0.05(0.1)$ is sufficient for data on pion (kaon) production.

Finally, conservation of the momentum of the fragmenting parton $f$ in the hadronization process is summarized by a sum rule stating that

$$
\sum_{H} \int_{0}^{1} d z z D_{i}^{H}\left(z, Q^{2}\right)=1
$$

i.e., each parton will fragment with $100 \%$ probability into some hadron $H$. Equation (5) is compatible with the evolution kernels in the $\overline{\mathrm{MS}}$ scheme, although not for each individual contribution $\int_{0}^{1} d z z D_{i}^{H}\left(z, Q^{2}\right)$. Of course, the sum rule (5) should be dominated, perhaps almost saturated, by the fragmentation into the lightest hadrons such as pions and kaons. The unstable small- $z$ behavior, however, prevents Eq. (5) from being a viable constraint in a global analysis. Only truncated moments $\int_{z_{\min }}^{1} d z z D_{i}^{H}\left(z, Q^{2}\right)$ are meaningful.

\section{B. Single-inclusive $e^{+} e^{-}$Annihilation}

The cross sections for the single-inclusive $e^{+} e^{-}$annihilation (SIA) into a specific hadron $H$,

$$
e^{+} e^{-} \rightarrow(\gamma, Z) \rightarrow H,
$$

at a center-of-mass system (c.m.s.) energy $\sqrt{s}$ and integrated over the production angle can be written as [29, 30]

$$
\frac{1}{\sigma_{t o t}} \frac{d \sigma^{H}}{d z}=\frac{\sigma_{0}}{\sum_{q} \hat{e}_{q}^{2}}\left[2 F_{1}^{H}\left(z, Q^{2}\right)+F_{L}^{H}\left(z, Q^{2}\right)\right] .
$$

The energy $E_{H}$ of the observed hadron scaled to the beam energy $Q / 2=\sqrt{s} / 2$ is denoted by $z \equiv 2 p_{H} \cdot q / Q^{2}=$ $2 E_{H} / \sqrt{s}$ with $Q$ being the momentum of the intermediate $\gamma$ or $Z$ boson.

$$
\sigma_{t o t}=\sum_{q} \hat{e}_{q}^{2} \sigma_{0}\left[1+\frac{\alpha_{s}\left(Q^{2}\right)}{\pi}\right]
$$

is the total cross section for $e^{+} e^{-} \rightarrow$ hadrons including its NLO $\mathcal{O}\left(\alpha_{s}\right)$ correction and $\sigma_{0}=4 \pi \alpha^{2}\left(Q^{2}\right) / s$. The sums in (7) and (8) run over the $n_{f}$ active quark flavors $q$, and the $\hat{e}_{q}$ are the corresponding appropriate electroweak charges (see App. A of Ref. [24] for details).

To NLO accuracy, the unpolarized "time-like" structure functions $F_{1}^{H}$ and $F_{L}^{H}$ in (7) are given by

$$
\begin{aligned}
2 F_{1}^{H}\left(z, Q^{2}\right)= & \sum_{q} \hat{e}_{q}^{2}\left\{\left[D_{q}^{H}\left(z, Q^{2}\right)+D_{\bar{q}}^{H}\left(z, Q^{2}\right)\right]\right. \\
& +\frac{\alpha_{s}\left(Q^{2}\right)}{2 \pi}\left[C_{q}^{1} \otimes\left(D_{q}^{H}+D_{\bar{q}}^{H}\right)\right. \\
& \left.\left.+C_{g}^{1} \otimes D_{g}^{H}\right]\left(z, Q^{2}\right)\right\} \\
F_{L}^{H}\left(z, Q^{2}\right)= & \frac{\alpha_{s}\left(Q^{2}\right)}{2 \pi} \sum_{q} \hat{e}_{q}^{2}\left[C_{q}^{L} \otimes\left(D_{q}^{H}+D_{\bar{q}}^{H}\right)\right. \\
& \left.+C_{g}^{L} \otimes D_{g}^{H}\right]\left(z, Q^{2}\right),
\end{aligned}
$$

with $\otimes$ denoting a standard convolution. The relevant NLO coefficient functions $C_{q, g}^{1, L}$ in the $\overline{\mathrm{MS}}$ scheme can be found in App. A of Ref. [24]. 
We note that the longitudinal structure function $F_{L}$ in Eq.(10) receives its leading nonzero (finite and scheme independent) contribution at $\mathcal{O}\left(\alpha_{s}\right)$. We treat, however, the $\mathcal{O}\left(\alpha_{s}\right)$ expressions in (10) as sub-leading (=NLO) in calculations of the total (longitudinal plus transverse) cross section (7). For predictions of only the longitudinal cross section at NLO, the $\mathcal{O}\left(\alpha_{s}^{2}\right)$ corrections [26] should be included. However, such measurements are not available for identified pions or kaons considered in this analysis.

\section{Semi-Inclusive Deep-Inelastic Scattering}

The cross section for the semi-inclusive deep-inelastic production of a hadron, $e N \rightarrow e^{\prime} H X$, is proportional to certain combinations of both the parton distributions of the nucleon $N$ and the fragmentation functions for the hadron $H$. It can be written in factorized form in a way very similar to the fully inclusive DIS case [24, 29, 30, 31]:

$$
\begin{aligned}
\frac{d \sigma^{H}}{d x d y d z_{H}}= & \frac{2 \pi \alpha^{2}}{Q^{2}}\left[\frac{\left(1+(1-y)^{2}\right)}{y} 2 F_{1}^{H}\left(x, z_{H}, Q^{2}\right)\right. \\
& \left.+\frac{2(1-y)}{y} F_{L}^{H}\left(x, z_{H}, Q^{2}\right)\right]
\end{aligned}
$$

with $x$ and $y$ denoting the usual DIS scaling variables $\left(Q^{2}=s x y\right)$, and where [29, 30] $z_{H} \equiv p_{H} \cdot p_{N} / p_{N} \cdot q$ with an obvious notation of the four-momenta, and with $-q^{2} \equiv Q^{2}$. Strictly speaking, Eq. (11) and the variable $z_{H}$ only apply to hadron production in the current fragmentation region. This is usually ensured by a cut $x_{F}>0$ on the Feynman-variable representing the fractional longitudinal c.m.s. momentum. If necessary, target fragmentation could be accounted for by transforming to the variable [31, 32] $z_{H} \rightarrow z \equiv \frac{E_{H}}{E_{N}(1-x)}$, the energies $E_{H}$, $E_{N}$ defined in the c.m.s. frame of the nucleon and the virtual photon, and by introducing the so-called "fracture functions" 32.

The structure functions $F_{1}^{H}$ and $F_{L}^{H}$ in (11) are given at NLO by

$$
\begin{aligned}
2 F_{1}^{H}\left(x, z_{H}, Q^{2}\right)= & \sum_{q, \bar{q}} e_{q}^{2}\left\{q\left(x, Q^{2}\right) D_{q}^{H}\left(z_{H}, Q^{2}\right)\right. \\
& +\frac{\alpha_{s}\left(Q^{2}\right)}{2 \pi}\left[q \otimes C_{q q}^{1} \otimes D_{q}^{H}\right. \\
& +q \otimes C_{g q}^{1} \otimes D_{g}^{H} \\
& \left.\left.+g \otimes C_{q g}^{1} \otimes D_{q}^{H}\right]\left(x, z_{H}, Q^{2}\right)\right\} \\
F_{L}^{H}\left(x, z_{H}, Q^{2}\right)= & \frac{\alpha_{s}\left(Q^{2}\right)}{2 \pi} \sum_{q, \bar{q}} e_{q}^{2}\left[q \otimes C_{q q}^{L} \otimes D_{q}^{H}\right. \\
& +q \otimes C_{g q}^{L} \otimes D_{g}^{H} \\
& \left.+g \otimes C_{q g}^{L} \otimes D_{q}^{H}\right]\left(x, z_{H}, Q^{2}\right),
\end{aligned}
$$

with the NLO $(\overline{\mathrm{MS}})$ coefficient functions $C_{i j}^{1, L}$ 24, 29, 30, 31.

In our global analysis of fragmentation functions we will make use of (preliminary) data for charged pion and charged kaon multiplicities taken by the HERMES experiment [18]. The multiplicities $\left(1 / N_{\text {DIS }}\right) d N^{H} / d z d Q^{2}$ are defined as the ratio of the semi-inclusive deep-inelastic scattering (SIDIS) cross section (11) in a certain bin of, say, $Q^{2}$ and $z$, to the totally inclusive DIS rate. The particular value of this data in the global analysis emerges from the sensitivity to individual quark and anti-quark flavors in the fragmentation process which is not accessible from $e^{+} e^{-}$annihilation.

\section{Hadron-Hadron Collisions}

The single-inclusive production of a hadron $H$ at high transverse momentum $p_{T}$ in hadron-hadron collisions is also amenable to QCD perturbation theory. Up to corrections suppressed by inverse powers of $p_{T}$, the differential cross section can be written in factorized form as [25, 33]

$$
E_{H} \frac{d^{3} \sigma}{d p_{H}^{3}}=\sum_{a, b, c} f_{a} \otimes f_{b} \otimes d \hat{\sigma}_{a b}^{c} \otimes D_{c}^{H},
$$

where the sum is over all contributing partonic channels $a+b \rightarrow c+X$, with $d \hat{\sigma}_{a b}^{c}$ the associated partonic cross section. $d \hat{\sigma}_{a b}^{c}$ can be expanded as a power series in the strong coupling $\alpha_{s}$ and the $\mathcal{O}\left(\alpha_{s}^{3}\right)$ NLO corrections are available [25, 33]. As always, the factorized structure (14) forces one to introduce into the calculation scales of the order of the hard scale in the reaction - but not specified further by the theory - that separate the short- and longdistance contributions. We have suppressed the explicit dependence on these renormalization and factorization scales in Eq. (14), for details, see, e.g., Ref. 25].

In studies and quantitative analyzes of hadronic cross sections, NLO corrections are of particular importance and generally indispensable in order to arrive at a firm theoretical prediction for (14). Since NLO corrections are known to be significant, LO approximations usually significantly undershoot the available data. In addition, hadronic reactions suffer from much enhanced theoretical uncertainties than the reactions described above due to the presence of more non-perturbative, scale dependent functions. The dependence on the unphysical factorization and renormalization scales can be only controlled and quantified at NLO (or beyond).

As will be discussed below, the special value of hadronic cross sections in a global analysis of fragmentation functions is their enhanced sensitivity to the gluon fragmentation function through the dominance of $g g \rightarrow$ $g X$ processes for hadrons produced at low-to-medium transverse momenta and their sensitivity to fragmentation at very high $z$. Charge separated data for $H=\pi^{ \pm}$ and $K^{ \pm}$provide additional information on the flavor separation of the $D_{i}^{H}$. 


\section{OUTLINE OF THE ANALYSIS}

In this Section, we outline the details of our analysis. More specifically, we discuss our choice of parametrization, the selection of data sets, treatment of experimental normalization uncertainties, and how we determine the parameters by means of a global $\chi^{2}$ minimization. We also briefly sketch how we make use of Mellin moments to include exact NLO expressions for the cross sections (7), (11), and (14) in our analysis and how we assess uncertainties in the extraction of fragmentation functions with the help of the Lagrange multiplier technique.

\section{A. Parametrization}

All recent analyses of fragmentation functions are based exclusively on SIA data [7, 8, 9, 10] and have chosen the most simple functional form $N_{i} z^{\alpha_{i}}(1-z)^{\beta_{i}}$ to parametrize the $D_{i}^{H}$ at some initial scale $\mu_{0}$ for the $Q^{2}$-evolution (2). The structure of the SIA cross section (77)-(10) allows to extract only information on $D_{q+\bar{q}}^{\pi^{+}+\pi^{-}}$ from data (similarly for kaons). Without assumptions it is impossible to distinguish "favored" or "valence" from "unfavored" or "sea" fragmentation, for instance, $D_{u}^{\pi^{+}}$ from $D_{\bar{u}}^{\pi^{+}}$where $\left|\pi^{+}\right\rangle=|u \bar{d}\rangle$. This is a serious limitation of all present analyses [7, 8, 9, 10], as the obtained fragmentation functions cannot be used to compare to a wealth of recent data on the production of charged pions and kaons in SIDIS [18] or proton-proton collisions [21]. In Ref. 7] a linear suppression factor $D_{\bar{u}}^{\pi^{+}} / D_{u}^{\pi^{+}}=(1-z)$ was assumed to break this "deadlock". This was later shown to be in fair agreement with charged pion multiplicities in SIDIS from HERMES [18] within a LO combined analysis of SIA and SIDIS data [34]; see also Fig. 4 and discussions below.

In our global analysis we will determine for the first time individual fragmentation functions for quark and anti-quarks for all flavors as well as gluons from data. To accommodate also the experimental information from lepton-nucleon and hadron-hadron scattering data, we adopt a somewhat more flexible input distribution than in $[7,8,9,10]$

$D_{i}^{H}\left(z, \mu_{0}\right)=\frac{N_{i} z^{\alpha_{i}}(1-z)^{\beta_{i}}\left[1+\gamma_{i}(1-z)^{\delta_{i}}\right]}{B\left[2+\alpha_{i}, \beta_{i}+1\right]+\gamma_{i} B\left[2+\alpha_{i}, \beta_{i}+\delta_{i}+1\right]}$,

where $B[a, b]$ represents the Euler Beta-function and $N_{i}$ is normalized such to represent the contribution of $D_{i}^{H}$ to the sum rule (5). A more restrictive initial parametrization with $\gamma_{i}=0$ in Eq. (15) would introduce artificial correlations between the behavior of fragmentation functions in different regions of $z$ obscuring also the assessment of uncertainties. We find that the extra term $\sim(1-z)^{\delta_{i}}$ in Eq. (15) considerably improves the quality of the global fit, closely related to the fact that the analysis of fragmentation functions is restricted to medium- to-large $z$. Accordingly, additional power terms in $z$, emphasizing the small $z$ region, have little or no impact on the fit and are not pursued further. The initial scale $\mu_{0}$ for the $Q^{2}$-evolution is taken to be $\mu_{0}=1 \mathrm{GeV}$ in our analysis.

Since the initial fragmentation functions (15) at scale $\mu_{0}$ should not involve more free parameters than can be extracted from data, we have to impose, however, certain relations upon the individual fragmentation functions for pions and kaons. We have checked in each case that relaxing these assumptions indeed does not significantly improve the $\chi^{2}$ of the fit of presently available data to warrant any additional parameters. In detail, for $\{u, \bar{u}, d, \bar{d}\} \rightarrow \pi^{+}$we impose isospin symmetry for the sea fragmentation functions, i.e.,

$$
D_{\bar{u}}^{\pi^{+}}=D_{d}^{\pi^{+}},
$$

but we allow for slightly different normalizations in the $q+\bar{q}$ sum:

$$
D_{d+\bar{d}}^{\pi^{+}}=N D_{u+\bar{u}}^{\pi^{+}} .
$$

For strange quarks it is assumed that

$$
D_{s}^{\pi^{+}}=D_{\bar{s}}^{\pi^{+}}=N^{\prime} D_{\bar{u}}^{\pi^{+}}
$$

with $N^{\prime}$ independent of $z$.

It is worth noticing that assuming $N=N^{\prime}=1[7,10]$ in Eqs. (17) and (18), respectively, SIA data alone allow to distinguish between favored and unfavored fragmentation functions in principle. We shall scrutinize the compatibility of these assumptions with SIDIS and hadronic scattering data in Sec. IVF At any rate, their impact on the assessment of uncertainties of fragmentation functions is highly non trivial.

For charged kaons we fit $D_{u+\bar{u}}^{K^{+}}$and $D_{s+\bar{s}}^{K^{+}}$independently to account for the phenomenological expectation that the formation of secondary $s \bar{s}$ pairs, which is required to form a $\left|K^{+}\right\rangle=|u \bar{s}\rangle$ from a $u$ but not from an $\bar{s}$ quark, should be suppressed. Indeed, we find from our fit, see Sec. IV below, that $D_{s+\bar{s}}^{K^{+}}>D_{u+\bar{u}}^{K^{+}}$in line with that expectation. For the unfavored fragmentation the data are unable to discriminate between flavors and, consequently, we assume that all distributions have the same functional form:

$$
D_{\bar{u}}^{K^{+}}=D_{s}^{K^{+}}=D_{d}^{K^{+}}=D_{\bar{d}}^{K^{+}} .
$$

We adopt the functional form (15) also for the fragmentation of heavy charm and bottom quarks into charged pions and kaons but setting $\gamma_{i}=0$. As in [7, 8, 9, 10] we assume that $D_{c}^{H}=D_{\bar{c}}^{H}$ and $D_{b}^{H}=D_{\bar{b}}^{H}$ for $H=\pi^{+}, K^{+}$. Heavy flavors are included discontinuously as massless partons in the evolution (2) above their $\overline{\mathrm{MS}}$ "thresholds", $Q=m_{c, b}$, with $m_{c, b}$ denoting the mass of the charm and bottom quark, respectively. This treatment of heavy flavors is very much at variance with heavy quark parton densities, where very elaborate schemes have been developed to properly include mass effects near threshold and 
to resum large logarithms $\sim \ln m_{c, b}^{2} / Q^{2}$ for $Q^{2} \gg m_{c, b}^{2}$. Only SIA data at $\sqrt{s} \gg m_{c, b}$ are sensitive, however, to charm and bottom fragmentation in the analysis. Neither the charged pion or kaon multiplicities in SIDIS nor hadron production data from proton-proton collisions at RHIC receive any noticeable contribution from heavy quark fragmentation. Therefore the massless approximation outlined above, also adopted in [7, 8, , 9, 10], appears to be sufficient for the time being. However, we note that a dynamical, parameter-free generation of the heavy flavor component to light meson fragmentation functions based on NLO matching conditions has been developed recently in 35]. This might prove to be a viable alternative to the presently adopted framework in the future.

Thus in total we have to determine 23 (24) parameters in the global $\chi^{2}$ analysis describing the hadronization of quarks and gluons into positively charged pions (kaons). Corresponding fragmentation functions into $\pi^{-}, K^{-}$are obtained as usual by charge conjugation and those for neutral pions by assuming $D_{i}^{\pi^{0}}=\left[D_{i}^{\pi^{+}}+D_{i}^{\pi^{-}}\right] / 2$.

We numerically solve the renormalization group equation in NLO

$$
\frac{d \alpha_{s}\left(\mu^{2}\right)}{d \ln \mu^{2}}=-\frac{\beta_{0}}{4 \pi} \alpha_{s}^{2}\left(\mu^{2}\right)-\frac{\beta_{1}}{(4 \pi)^{2}} \alpha_{s}^{3}\left(\mu^{2}\right)
$$

with $\beta_{0}=11-2 n_{f} / 3$ and $\beta_{1}=102-38 n_{f} / 3$ to determine the running of the strong coupling. The number of active flavors $n_{f}$ is increased upon crossing the heavy flavor thresholds at $\mu^{2}=m_{c}^{2}$ and $\mu^{2}=m_{b}^{2}$ for which we choose [36] $m_{c}=1.43 \mathrm{GeV}$ and $m_{b}=4.3 \mathrm{GeV}$. We specify $\alpha_{s}$ in the solution of (20) for $n_{f}=4$ by adopting [36] $\Lambda_{\mathrm{QCD}}^{\left(n_{f}=4\right)}=$ $334 \mathrm{MeV}$. For our leading order (LO) analysis we use 37] $\Lambda_{\mathrm{QCD}}^{\left(n_{f}=4\right)}=220 \mathrm{MeV}$ and, of course, set $\beta_{1}=0$ in Eq. (20).

\section{B. Selection of Data Sets}

The parameters describing the fragmentation functions for pions and kaons at scale $\mu_{0}$ in Eq. (15) are determined by a standard $\chi^{2}$ minimization for $N$ data points, where

$$
\chi^{2}=\sum_{i=1}^{N} \frac{\left(T_{i}-E_{i}\right)^{2}}{\delta E_{i}^{2}}
$$

$E_{i}$ is the measured value of a given observable, $\delta E_{i}$ the error associated with this measurement, and $T_{i}$ is the corresponding theoretical estimate for a given set of parameters in (15). Since the full error correlation matrices are not available for most of the data entering the global analysis, we take, as usual [7, 8, 9, 10], the statistical and systematical errors in quadrature in $\delta E_{i}$.

In (21) we use charged pion and kaon production data in SIA from TPC [15] at $\sqrt{s}=29 \mathrm{GeV}$, SLD [16], ALEPH [11], DELPHI [12], and OPAL [13], all at $\sqrt{s}=M_{Z}$. To further constrain fragmentation functions through scale evolution, we also use data from TASSO [17] at intermediate c.m.s. energies of $\sqrt{s}=33$ and $44 \mathrm{GeV}$, which suffer, however, from rather large experimental uncertainties as compared to the other SIA data listed above. Other measurements of SIA 38] have too large experimental uncertainties and hence are not used in our analysis. Because of the conceptual problem with fragmentation functions at small $z$ outlined in Sec. IIA, the cut $z_{\min }=0.05(0.1)$ is imposed for all pion (kaon) data sets.

Besides these fully inclusive measurements also "flavor tagged" SIA results are available, where the quark flavor refers to the primary $q \bar{q}$ pair created by the intermediate photon or $Z$-boson. ALEPH [11], DELPHI 12], and TPC [15] provide tagged results distinguishing between the sum of light $u, d, s$ quarks, charm, and bottom events. This information is of particular value for the flavor decomposition, as the fully inclusive, "untagged" data mainly constrain the flavor singlet combination $D_{\Sigma}^{H}(z)$ on the $Z$-resonance due overwhelming statistical precision of the LEP and SLD data and the fact that $\hat{e}_{u}^{2} \simeq \hat{e}_{d}^{2}$ at $Q=M_{Z}$. On the downside, flavor tagged results can neither be measured directly nor can they be unambiguously interpreted and calculated in pQCD. Flavor enriched samples are unfolded based on Monte-Carlo simulations estimating the flavor composition of the data sets. For heavy flavor tagged data, a further complication arises due to possible contaminations by weak decay channels. In our analysis, we obtain the corresponding theoretical results $T_{i}$ by summing in Eqs. (7)-(10) only over those flavors which are tagged experimentally. At NLO this accounts for gluon radiation as well as the possibility that not the original (anti-)quark but the radiated gluon produces the observed hadron.

In addition to the flavor tagged results just discussed, OPAL 14 has presented fully flavor separated "data" in terms of "probabilities" $\eta_{i}^{H}\left(x_{p}, s\right)$ for a quark flavor $i=q+\bar{q}$ to produce a "jet" containing the hadron $H$ with a momentum fraction $z$ larger than $x_{p}$. Needless to say, that these results are even more difficult to interpret within $\mathrm{pQCD}$ beyond the LO and should not be taken to literally. To take this into account we assign an up to $10 \%$ normalization uncertainty to the OPAL tagging probabilities $\eta_{i}^{H}$ [14] in the fit. Nevertheless some tension with other data sets remains, in particular for $\eta_{c}^{H}$ and $\eta_{b}^{H}$, as will be discussed below. In our analysis we interpret the OPAL results as

$$
\eta_{i}^{H}\left(x_{p}, Q=M_{Z}\right)=\left.\int_{x_{p}}^{1} d z \frac{1}{\sigma_{t o t}} \frac{d \sigma^{H}}{d z}\right|_{i=q},
$$

where the subscript $i=q$ denotes that in Eqs. (7)-(10) all sums only include the specific quark flavor $i$.

To further constrain the fragmentation of different flavors, as well as too separate favored (valence) and unfavored (sea) fragmentation, we include experimental information from SIDIS, see Sec.IIC. More specifically, we make use of (preliminary) charged pion and kaon multiplicities from the HERMES experiment [18]. These data also provide an important consistency check of the flavor separation obtained from flavor tagged SIA "data", as 
well as of pQCD scale evolution (2) since they refer to much lower scales $\mu \simeq Q=1 \div 3 \mathrm{GeV} \ll M_{Z}$.

In the $\chi^{2}$ minimization we have to account for the fact that the SIDIS data are taken in certain bins of $z$ and $Q^{2}$ [18], whereas the theoretical estimates (11) are computed for the center of each bin. We estimate the corresponding uncertainty as the maximal variation of the cross section within each particular bin, see Figs. 4 and 13 below, and add it in quadrature to $\delta E_{i}$ in Eq. (21). In case of charged kaon multiplicities, we allow for an additional $5 \%$ uncertainty to account for a possible inadequacy of the massless approximation.

A wealth of new data on single-inclusive hadron production from RHIC experiments [19, 20, 21, 22] have also been included in our global analysis. These encompass the $p_{T}$ spectrum of neutral pions at central rapidities $|\eta| \leq 0.35$ by PHENIX [19] and at three different forward rapidities $\langle\eta\rangle=3.3,3.8$, and 4.0 by STAR [20]. For the latter we exclude the most forward bin, $\langle\eta\rangle=4.0$, from the fit as it has large theoretical uncertainties due the small $p_{T}$ values probed. BRAHMS has very recently published $p_{T}$ spectra for identified charged pions and kaons at two values of (forward) rapidities $\eta=2.95$ and 3.3 [21] of which we use only the former in the fit for similar reasons as above for STAR. In addition, there are data on $K_{S}^{0}$ production at central rapidities $|\eta| \leq 0.5$ from STAR [22]. To accommodate the $K_{S}^{0}$ data in the fit, we assume that $K_{S}^{0}=\left(K^{+}+K^{-}\right) / 2$ with $u \rightarrow K^{+}$and $d \rightarrow K^{+}$ fragmentation functions interchanged.

For all hadronic data from RHIC, an additional $5 \%$ error is assigned in quadrature to $\delta E_{i}$ in evaluations of $\chi^{2}$ in Eq. (21) as a rather conservative estimate of the theoretical uncertainties related to the choice of the factorization and renormalization scales in (14).

To ease possible tensions between certain data sets, we allow the data to "float" within the normalization uncertainties quoted by each experiment. More precisely, in addition to the $\mathcal{O}(20)$ parameters describing the fragmentation functions in (15), we also fit a set of relative normalization factors for each experiment in the $\chi^{2}$ minimization to determine the optimum fit. We note that the possibility of normalization uncertainties has been not addressed in all previous analysis of SIA data 7, , 8, [9, 10].

\section{Mellin Technique}

The integro-differential evolution equations (2) can be straightforwardly solved analytically in Mellin $n$-moment space along the lines described, e.g., in Ref. [39]. The Mellin moments of, for instance, the fragmentation functions $D_{i}^{H}\left(z, Q^{2}\right)$, are defined as

$$
D_{i}^{H}\left(n, Q^{2}\right) \equiv \int_{0}^{1} d z z^{n-1} D_{i}^{H}\left(z, Q^{2}\right),
$$

and can be expressed in terms of Euler Beta functions for our ansatz (15) at scale $\mu_{0}$. The relevant moments of the evolution kernels $P_{i j}^{(T)}(n)$ are given in [40]. The evolved fragmentation functions in $z$-space are re-obtained by an inverse Mellin transform given by

$$
D_{i}^{H}\left(z, Q^{2}\right)=\frac{1}{2 \pi i} \int_{\mathcal{C}_{n}} d n z^{-n} D_{i}^{H}\left(n, Q^{2}\right),
$$

where $\mathcal{C}_{n}$ denotes an appropriately chosen contour in the complex $n$ plane.

The property that numerically very time-consuming convolutions in $z$-space factorize into simple products under Mellin moments makes them also an ideal tool to compute cross sections. Also "plus distributions", which regularize singularities as $z \rightarrow 1$, are much easier to handle. For the SIA cross section (7) - (10) the virtue of Mellin moments is immediately obvious since they can be taken analytically for the hard scattering coefficient functions $C_{q, g}^{1, L}$ and can be found in Ref. [40]. We note that also the "tagging probabilities" $\eta_{i}^{H}$ in Eq. (22) as obtained by OPAL [14] can be straightforwardly computed in Mellin moment space

$$
\eta_{i}^{H}\left(x_{p}, Q=M_{Z}\right)=\left.\frac{1}{2 \pi i} \int_{\mathcal{C}_{n}} d n \frac{1-x_{p}^{(1-n)}}{1-n} \frac{1}{\sigma_{t o t}} \frac{d \sigma^{H}}{d n}\right|_{i=q},
$$

where $\left.\frac{1}{\sigma_{t o t}} \frac{d \sigma^{H}}{d n}\right|_{i=q}$ denote the Mellin moments of the SIA cross section (7) - (10) for a single flavor $i=q$.

The direct use of the SIDIS cross section (11)-(13) in our global analysis would be rather time-consuming and awkward though not impossible since the partonic coefficient functions $C_{i j}^{1, L}$ are still fairly simple. Again, transforming Eqs. (11)- (13) to Mellin space is much more appropriate in extensive numerical analyses. As for SIA, the Mellin moments of the coefficient functions can be taken completely analytically and can be found in [29, 41]. Since the $C_{i j}^{1, L}$ depend both on $x$ and $z$, a double Mellin transform is required for SIDIS:

$$
C_{i j}^{(1, L)}(n, m) \equiv \int_{0}^{1} d x x^{n-1} \int_{0}^{1} d z z^{m-1} C_{i j}^{(1, L)}(x, z),
$$

where the dependence on the factorization and renormalization scales is suppressed in (26). Upon combining the $C_{i j}^{(1, L)}(n, m)$ with the appropriate $n$ and $m$ moments of the evolved parton densities $f_{i}\left(n, Q^{2}\right)$ and fragmentation functions $D_{j}^{H}\left(m, Q^{2}\right)$, respectively, the SIDIS cross section is obtained by a numerical fast double inverse Mellin transform as described in [41].

For the much more complex and lengthy partonic hard cross sections entering a calculation of hadronic cross sections, Eq. (14), at NLO accuracy, Mellin moments can no longer be computed analytically. Nevertheless it is in the analysis of hadron-hadron collision data where the Mellin moment technique exhibits its full potential and usefulness [41, 42]. The crucial, but simple, "trick" in applying Mellin moments to Eq. (14) is to express the fragmentation functions $D_{c}^{H}(z)$ by their Mellin inverses $D_{c}^{H}(n)$ 
defined in Eq. (24). One subsequently interchanges integrations and arrives schematically at

$$
\begin{aligned}
E_{H} \frac{d^{3} \sigma}{d p_{H}^{3}}= & \frac{1}{2 \pi i} \sum_{c} \int_{\mathcal{C}_{n}} d n D_{c}^{H}(n) \\
& \times\left[\sum_{a b} f_{a} \otimes f_{b} \otimes d \hat{\sigma}_{a b}^{c} \otimes z^{-n}\right] .
\end{aligned}
$$

One can now pre-calculate the quantities $d \tilde{\hat{\sigma}}_{a b}^{c}(n) \equiv$ $\sum_{a b} f_{a} \otimes f_{b} \otimes d \hat{\sigma}_{a b}^{c} \otimes z^{-n}$ in (27), which do not depend on the fragmentation functions $D_{c}^{H}(n)$, prior to the fit for each contributing combination of single-inclusive subprocesses producing a certain parton $c$ and in each experimental bin. We emphasize that in this way all the tedious and time-consuming integrations are already dealt with.

The inverse Mellin transformation, which finally links the moments of the fitted fragmentation functions with the pre-calculated $d \tilde{\hat{\sigma}}_{a b}^{c}(n)$, of course still needs to be performed in each step of the fitting procedure. However, the integration over $n$ is extremely fast to perform by choosing the values for $n$ on the contour $\mathcal{C}_{n}$ in (27) simply as the supports for a Gaussian integration. The point here is that the integrand in $n$ falls off very rapidly as $|n|$ increases along the contour for two reasons: first, each fragmentation function is expected to fall off at least as a power $(1-z)^{\beta}, \beta \geq 1$, at large $z$, which in moment space converts into a fall-off of $\sim 1 / n^{\beta+1}$ or higher. Secondly, we may choose a contour $\mathcal{C}_{n}$ that is bent by an angle with respect to the standard vertical direction such that for large $|n|,(z)^{-n}$ decreases exponentially along the contour [41]. This greatly improves the numerical convergence of the calculation of the $d \tilde{\hat{\sigma}}_{a b}^{c}(n)$ in Eq. (27) and also gives them a rapid fall-off at large arguments. We note that for all practical purposes of our global analysis between 64 and $92 n$ moments of $d \tilde{\hat{\sigma}}_{a b}^{c}(n)$ are sufficient to reproduce the cross section (14) to an accuracy of much better than $1 \%$ for all data points used in the fit.

The crucial asset of the Mellin method is the speed at which one can calculate the full hadronic cross section at NLO without approximations, once the moments $d \tilde{\hat{\sigma}}_{a b}^{c}(n)$ have been pre-calculated. To give an example, a full NLO computation of all 78 data points from RHIC used in the analysis of the pion fragmentation functions takes much less than 0.1 second as compared to several minutes using Eq. (14) directly. Since a few thousand evaluations of each data point are required in course of the $\chi^{2}$ minimization, this clearly shows the value of using Mellin moments.

\section{Uncertainties: Lagrange Multiplier Technique}

The most difficult but crucial issue to be addressed in a global analysis is the estimate of the uncertainties in the extraction of the individual fragmentation functions $D_{i}^{H}$. Without a proper assessment of errors, any interpretation of the results of the fit or predictions for observables based on the fitted $D_{i}^{H}$ are incomplete and perhaps even misleading. Uncertainties in global analyses have been thoroughly studied in the context of unpolarized parton distributions (PDFs) [23, 36, 43], where the number and precision of the data available is much more significant. A reliable estimate of the errors arising from PDFs in predictions for observables related to, e.g., new physics or Higgs boson searches at CERN-LHC is of utmost importance.

The possible sources of uncertainties for parton densities or fragmentation functions can be classified into those associated with experimental errors on the data, and those related to theoretical or phenomenological assumptions in the global fitting procedure. The latter include, for example, higher order QCD effects in the analyzed cross sections and their scale dependence, the particular choice of the parametric form of the distributions at the initial scale, and other model assumptions such as flavor and charge conjugation symmetries. Clearly, while the first category is usually under control, the second one is particularly difficult to quantify.

Many strategies have been conceived and explored in order to assess the uncertainties of PDFs and their propagation to observables, specially those associated with experimental errors in the data. These include the "Hessian approach" 23], which assumes that the deviation in $\chi^{2}$ for the global fit is quadratic in the parameters specifying the input PDFs away from their optimum fit values. Then one propagates these uncertainties of PDFs linearly to observables. Alternatively, the "Lagrange multiplier method" 23] probes the uncertainty in any observable or quantity of interest much more directly. It relates the range of variation of one or more physical observables dependent upon PDFs to the variation in the $\chi^{2}$ used to judge the goodness of the fit to data. Specifically, it can be implemented by minimizing the function

$$
\Phi\left(\lambda_{i},\left\{a_{j}\right\}\right)=\chi^{2}\left(\left\{a_{j}\right\}\right)+\sum_{i} \lambda_{i} O_{i}\left(\left\{a_{j}\right\}\right)
$$

with respect to the set of parameters $\left\{a_{j}\right\}$ describing the PDFs, for fixed values of the Lagrange multipliers $\lambda_{i}$. Each one of the parameters $\lambda_{i}$ is related to an observable $O_{i}$ depending on $\left\{a_{j}\right\}$. The choice $\lambda_{i}=0$ in (28) corresponds to the optimum global fit $\left\{a_{j}\right\}$, for which $\chi^{2}\left(\left\{a_{j}\right\}\right) \equiv \chi_{0}^{2}$ and $O_{i}\left(\left\{a_{j}\right\}\right) \equiv O_{i}^{0}$. Minimizing $\Phi\left(\lambda_{i},\left\{a_{j}\right\}\right)$ for $\lambda_{i} \neq 0$ deteriorates the quality of the fit to data and other values for the observable $O_{i}\left(\left\{a_{j}\right\}\right)$ are found from the set of newly fitted parameters $\left\{a_{j}\right\}$. From a series of global fits for different values of $\lambda_{i}$, the $\chi^{2}\left(\left\{a_{j}\right\}\right)$ profile depending on different values of $O_{i}$ can be mapped out. In other words, this tell us by how much the fit to data deteriorates if we force the PDFs to yield a prediction for an observable different to the one obtained with the best fit $O_{i}^{0}$.

The value and practical feasibility of the Lagrange multiplier technique has been demonstrated not only for the highly sophisticated global analyses of unpolarized PDFs [23], but also in case of polarized PDFs [44]. In Sec.IVF. 
we will show that the same holds for the analysis of fragmentation functions. Here, the limitations due to the available data are in some sense similar to those we encounter for polarized PDFs: the bulk of the data (SIA and spin-dependent DIS in case of fragmentation functions and polarized PDFs, respectively) neither determine the gluon well nor allow for a reliable flavor separation. In both types of analysis, SIDIS and hadronic data provide invaluable constraints on the parameter space describing the input densities.

In an ideal situation, where every source of uncertainty is well understood and fully accounted for, all $\chi^{2}$ profiles, including those for the parameters $\left\{a_{j}\right\}$ of the fit, would be parabolic, and the 1- $\sigma$ uncertainty for any observable would correspond to a change in $\chi^{2}$ by one unit, i.e., $\Delta \chi^{2}=1$. This is, of course, rarely the case, and in order to account for missing correlated experimental errors or theoretical uncertainties in global analysis it is customary to consider instead of $\Delta \chi^{2}=1$ a $2 \div 5 \%$ variation in $\chi^{2}$ as a more conservative estimate of the range of uncertainty [23, 36, 43, 44].

In addition to the possibility of assessing the uncertainties of parameters $a_{j}$ or observables $O_{i}\left(\left\{a_{j}\right\}\right)$, the Lagrange multiplier method allows to elucidate the role of each subset of data included in the fit in constraining a certain quantity. One just needs to determine the shape and variation of the partial contribution $\Delta \chi_{n}^{2}$ of a particular subset $n$ of data to the total $\chi^{2}$ as the observable changes depending on the Lagrange multipliers. When a given subset of data can by itself determine, say, a given observable $O_{i}\left(\left\{a_{j}\right\}\right)$, the profile of $\Delta \chi_{n}^{2}$ w.r.t. $O_{i}\left(\left\{a_{j}\right\}\right)$ is expected to be roughly parabolic, with a minimum close to the "preferred" value $O_{i}^{0}$ determined by the optimum global fit. However, when a given subset of data does not fully constrain the observable $O_{i}\left(\left\{a_{j}\right\}\right)$, its profile w.r.t. $O_{i}\left(\left\{a_{j}\right\}\right)$ is either flat or increases (decreases) monotonically without minimum in the range of variation of the observable. In general, constraints on $O_{i}\left(\left\{a_{j}\right\}\right)$ in a global analysis result from the subtle interplay of several subsets of data. The combination of the different partial contributions to $\chi^{2}$, even of those that by themselves do not show a minimum, define the final $\chi^{2}$ profile and the best fit value $O_{i}^{0}$, thereby highlighting the complementary nature of a global analysis. For our fragmentation functions this will be illustrated in detail in Sec. IVF

\section{RESULTS}

In this Section we discuss in detail the results of our global analysis of fragmentation functions for pions and kaons. We present the parameters of the optimum fits describing the $D_{i}^{\pi^{+}, K^{+}}$at the input scale $\mu_{0}$, compare to the data used in the analysis, and give $\chi^{2}$ values for each individual set of data used. Detailed comparisons are made with the results obtained in the analyses of SIA data in Refs. [7] and [9], in the following labeled as KRE and AKK, respectively. Even though we are mainly inter- ested in a precise extraction of fragmentation functions at NLO accuracy, we also briefly present corresponding results of a global analysis performed at LO approximation. The significantly better $\chi^{2}$ of the NLO sets highlights the importance of the NLO corrections and the limitations of a LO analysis. Nevertheless, our LO sets should be used in calculations of observables where NLO corrections are not available, or in event generators limited to LO accuracy.

\section{A. NLO analysis of pion fragmentation functions}

From a first glance at Figures 1]8, one immediately notices the remarkable agreement between our new NLO fit and data. Experimental results for inclusive hadron production in SIA and proton-proton collisions span several orders of magnitude, and the energy scale of the different processes ranges from $1 \mathrm{GeV}$ to the mass of the $Z$-boson. This strongly supports the underlying theoretical framework outlined in Sec. III in particular the fundamental notions of factorization and universality for fragmentation functions.

Existing sets of NLO pion fragmentation functions [7, 9] also give a nice overall description of the SIA data included in these analyses, as indicated in Figs. 1,3. They fail, however, to satisfactorily reproduce charged pion production data obtained in SIDIS and in protonproton collisions, Figs. 4and 5, respectively. In addition, estimates for neutral pion production rates in protonproton collisions based on KRE [7] or AKK [9] fragmentation function differ substantially as can be seen in Figs. 6 and 7 On the contrary, our new set of NLO fragmentation functions gives, for the first time, a nice global description of hadron production data in electronpositron, lepton-nucleon, and hadron-hadron scattering, which constitutes a significant and necessary improvement.

The most significant difference between our NLO global analysis and previous extractions of $D_{i}^{\pi}$ in $[7, \underline{7}, \underline{9}$, 10] is the fact that we can now determine most aspects of the fragmentation functions from data rather than being forced to make assumptions due to the insufficient information contained in the SIA data alone. We find that, in particular, the extra freedom regarding flavor symmetry (or the lack thereof) as introduced in Eqs. (17) and (18) allows us to reproduce the charged pion data shown in Figs. 4 and 5 . In Table 1 we give the set of parameters specifying the optimum fit of pion fragmentation functions at NLO accuracy in Eq. (15) at our input scale $\mu_{0}=1 \mathrm{GeV}$ for the light flavors and the gluon, and at $\mu_{0}=m_{c}=1.43 \mathrm{GeV}$ and $\mu_{0}=m_{b}=4.3 \mathrm{GeV}$, for charm and bottom fragmentation, respectively. As can be inferred from there, the outcome of the global analysis deviates from the symmetry assumptions 7,10$] N=1$ and $N^{\prime}=1$ in Eqs.(17) and (18) by more than $10 \%$ and $20 \%$, respectively.

Another crucial asset of our analysis is the enhanced 

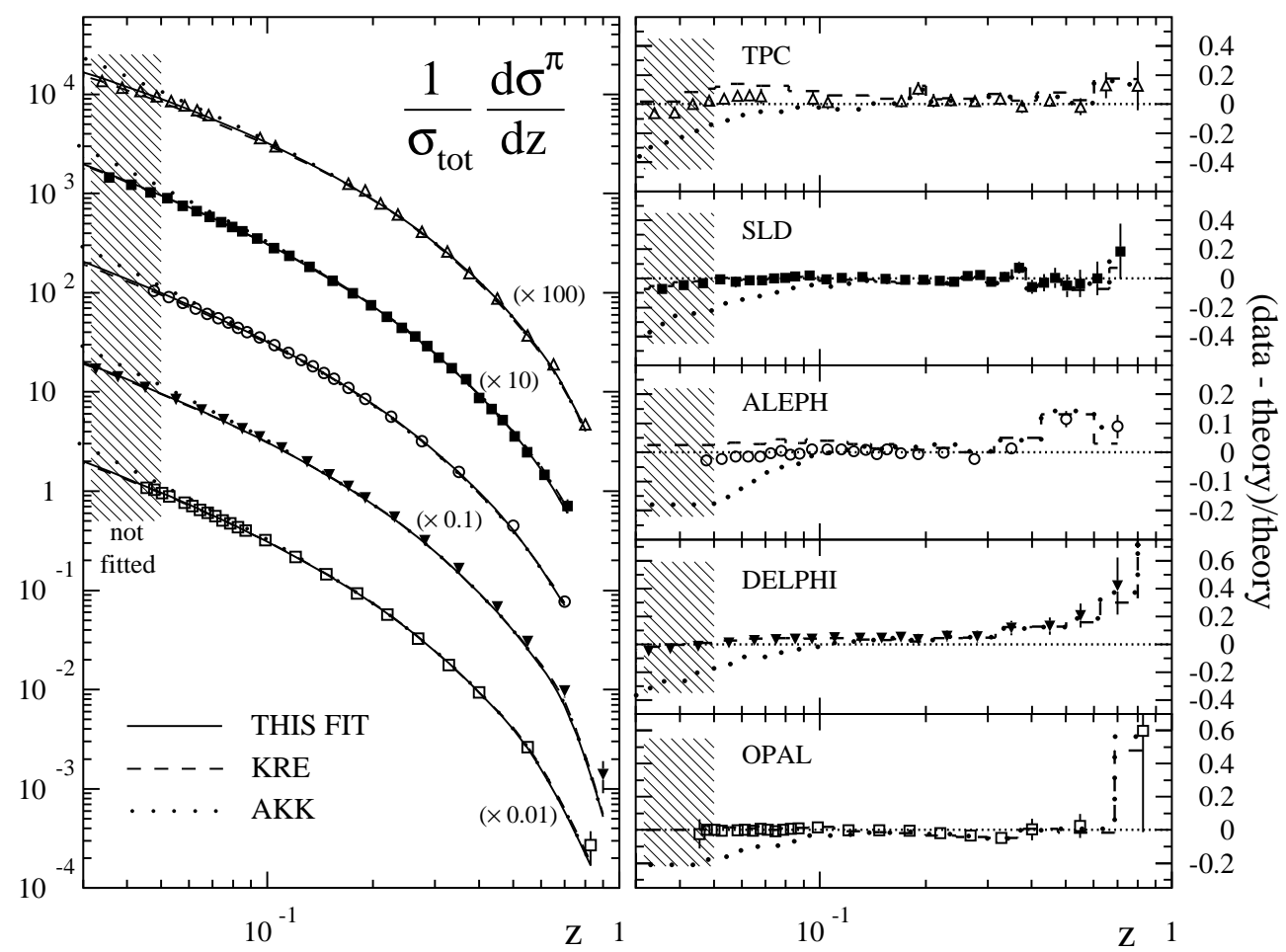

FIG. 1: L.h.s. comparison of our NLO results for $\left(1 / \sigma_{t o t}\right) d \sigma^{\pi} / d z$ according to Eq. (7) with the data sets for inclusive pion production in SIA used in the fit, see Tab. [I] R.h.s. "(data-theory)/theory" for our NLO results for each of the data sets. Also shown are the results obtained with the KRE [7] and AKK [9] parameterizations, dashed and dotted lines, respectively.
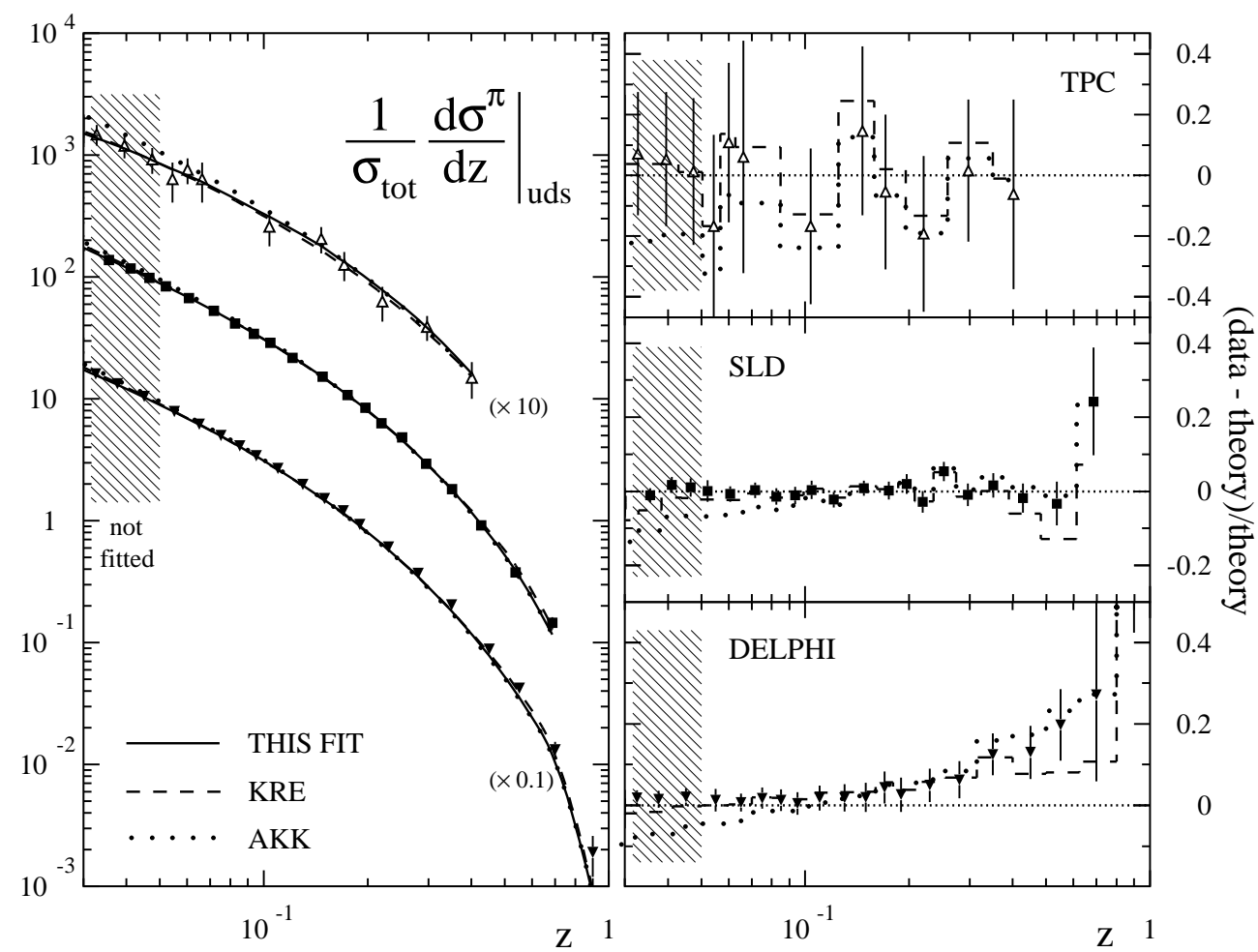

FIG. 2: Same as in Fig. 1 but now for light quark ("uds") tagged cross sections. 
TABLE I: Parameters describing the NLO fragmentation functions for positively charged pions, $D_{i}^{\pi^{+}}\left(z, \mu_{0}\right)$, in Eq. (15) at the input scale $\mu_{0}=1 \mathrm{GeV}$. Inputs for the charm and bottom fragmentation functions refer to $\mu_{0}=m_{c}=1.43 \mathrm{GeV}$ and $\mu_{0}=m_{b}=4.3 \mathrm{GeV}$, respectively.

\begin{tabular}{cccccc}
\hline \hline flavor $i$ & $N_{i}$ & $\alpha_{i}$ & $\beta_{i}$ & $\gamma_{i}$ & $\delta_{i}$ \\
\hline$u+\bar{u}$ & 0.345 & -0.015 & 1.20 & 11.06 & 4.23 \\
$d+\bar{d}$ & 0.380 & -0.015 & 1.20 & 11.06 & 4.23 \\
$\bar{u}=d$ & 0.115 & 0.520 & 3.27 & 16.26 & 8.46 \\
$s+\bar{s}$ & 0.190 & 0.520 & 3.27 & 16.26 & 8.46 \\
$c+\bar{c}$ & 0.271 & -0.905 & 3.23 & 0.00 & 0.00 \\
$b+\bar{b}$ & 0.501 & -1.305 & 5.67 & 0.00 & 0.00 \\
$g$ & 0.279 & 0.899 & 1.57 & 20.00 & 4.91 \\
\hline \hline
\end{tabular}

flexibility of the initial light quark and gluon fragmentation functions as a function of $z$ in Eq. (15) as compared to the standard three parameter form used so far [7, 8, 9, 10]. This is not only indispensable to accommodate SIDIS and hadron-hadron scattering data but even somewhat improves the quality of the fit to the SIA data. Indeed, upon closer examination of Figs. 1,3, in particular the "(data-theory)/theory" insets for each data set on the right-hand side (r.h.s.) of each plot, one finds a slightly improved overall agreement with data as compared to the, still excellent, one for KRE and AKK; see, for instance, the TPC or ALEPH data in Fig. 1 .

Noticeable is also that our fit follows the trend of the data even below the $z$ values included in the analysis. As for KRE [7], all data below $z_{\min }=0.05$ are not taken into account in the $\chi^{2}$ minimization to ensure that the possible impact of small- $z$ resummations or hadron mass effects, see Sec. IIA is negligible. In contrast, the agreement with data rapidly deteriorates for AKK [9] immediately below $z=0.1$, from which they choose to exclude data from the fit. This might be linked with the less flexible functional form for the fragmentation functions.

In Tab. II we list all data sets included in our global analysis as discussed in Sec. IIIB and give the individual $\chi^{2}$ values for each set. We note that quoted $\chi^{2}$ values are based only on fitted data points, i.e., $z>0.05$ for SIA, and include normalization uncertainties determined for each experiment in the fit. Allowing for relative normalizations in a global analysis within the range quoted by each experiment is a common tool to ease possible tensions between certain data sets. Indeed we find that the global fit considerably improves after taking into account normalization "shifts".

In spite of the nice "visual" agreement between the fit and data found in Figs. 1,8, the total $\chi^{2}$ of 843.7 units in Tab. II appears to be fairly large in view of the roughly 400 data points fitted. For the SIA data, the large $\chi^{2}$ can be pinpointed to only very few data points. Due to extremely high precision of the data on the $Z$-resonance, any deviation between data and theory is strongly penalized in the $\chi^{2}$ evaluation and results in an overall $\chi^{2}$
TABLE II: Data used in the NLO global analysis of pion fragmentation functions, the individual $\chi^{2}$ values for each set, the fitted normalizations, and the total $\chi^{2}$ of the fit.

\begin{tabular}{|c|c|c|c|c|}
\hline experiment & $\begin{array}{l}\text { data } \\
\text { type }\end{array}$ & $\begin{array}{c}\text { rel. norm. } \\
\text { in fit }\end{array}$ & $\begin{array}{c}\text { data points } \\
\text { fitted }\end{array}$ & $\chi^{2}$ \\
\hline \multirow[t]{4}{*}{ TPC [15] } & incl. & 0.94 & 17 & 18.5 \\
\hline & "uds tag" & 0.94 & 9 & 1.9 \\
\hline & "c tag" & 0.94 & 9 & 5.7 \\
\hline & "b tag" & 0.94 & 9 & 7.4 \\
\hline \multirow[t]{2}{*}{ TASSO [17] } & incl. $(34 \mathrm{GeV})$ & 0.94 & 11 & 30.1 \\
\hline & incl. $(44 \mathrm{GeV})$ & 0.94 & 7 & 20.5 \\
\hline \multirow[t]{4}{*}{ SLD [16] } & incl. & 1.008 & 28 & 14.0 \\
\hline & "uds tag" & 1.008 & 17 & 11.6 \\
\hline & "c tag" & 1.008 & 17 & 11.1 \\
\hline & "b tag" & 1.008 & 17 & 33.2 \\
\hline ALEPH [11] & incl. & 0.97 & 22 & 38.3 \\
\hline \multirow[t]{3}{*}{ DELPHI [12] } & incl. & 1.0 & 17 & 42.3 \\
\hline & "uds tag" & 1.0 & 17 & 26.4 \\
\hline & "b tag" & 1.0 & 17 & 42.8 \\
\hline \multirow[t]{6}{*}{ OPAL $[13,14]$} & incl. & 1.0 & 21 & 9.2 \\
\hline & "u tag" & 1.10 & 5 & 11.8 \\
\hline & "d tag" & 1.10 & 5 & 9.0 \\
\hline & "s tag" & 1.10 & 5 & 49.8 \\
\hline & "c tag" & 1.10 & 5 & 38.3 \\
\hline & "b tag" & 1.10 & 5 & 73.0 \\
\hline \multirow[t]{2}{*}{ HERMES [18] } & $\pi^{+}$ & 1.03 & 32 & 67.4 \\
\hline & $\pi^{-}$ & 1.03 & 32 & 120.8 \\
\hline PHENIX [19] & $\pi^{0}$ & 1.09 & 23 & 76.4 \\
\hline \multirow[t]{2}{*}{ STAR [22] } & $\pi^{0},\langle\eta\rangle=3.3$ & 1.05 & 4 & 3.4 \\
\hline & $\pi^{0},\langle\eta\rangle=3.7$ & 1.05 & 5 & 9.8 \\
\hline \multirow[t]{2}{*}{ BRAHMS [21] } & $\pi^{+},\langle\eta\rangle=2.95$ & 1.0 & 18 & 28.2 \\
\hline & $\pi^{-},\langle\eta\rangle=2.95$ & 1.0 & 18 & 43.0 \\
\hline TOTAL: & & & 392 & 843.7 \\
\hline
\end{tabular}

per degree of freedom which is rather large. This is a common "characteristic" of all extractions of fragmentation function made so far [7, 8, 9, 10]. We also wish to point out, that there is a tension between the behavior of the DELPHI data at large $z$ and those of all the other data sets at $Q=M_{Z}$ which cannot be resolved by the fit, see Figs. 1,3. The in general larger $\chi^{2}$ values of the heavy flavor, in particular bottom quark, tagged SIA cross sections in Fig. 3 might be related to some extent to contaminations from weak decays.

We will further scrutinize the quality of the fit to the SIDIS and hadronic data in the following. As illustrated in Fig. 4, the agreement between the (preliminary) charged pion multiplicities in SIDIS from the HERMES experiment [18] and the results of our fit is remarkably good. The theoretical estimates for the multiplicities are computed using PDFs from Ref. [36] as input in Eq. (12), although no significant differences are found using other 

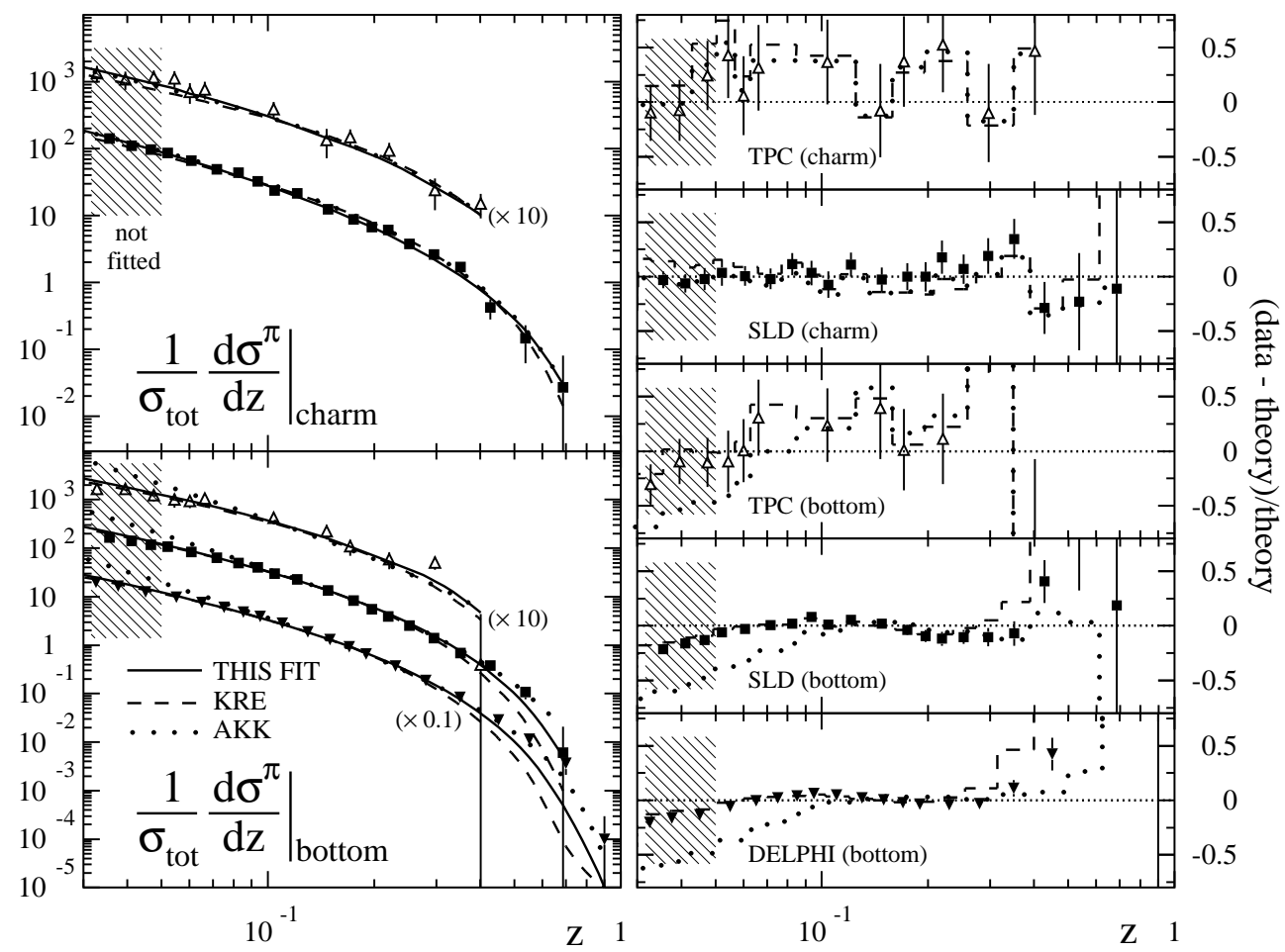

FIG. 3: Same as in Fig. 1 but now for charm and bottom quark tagged cross sections.
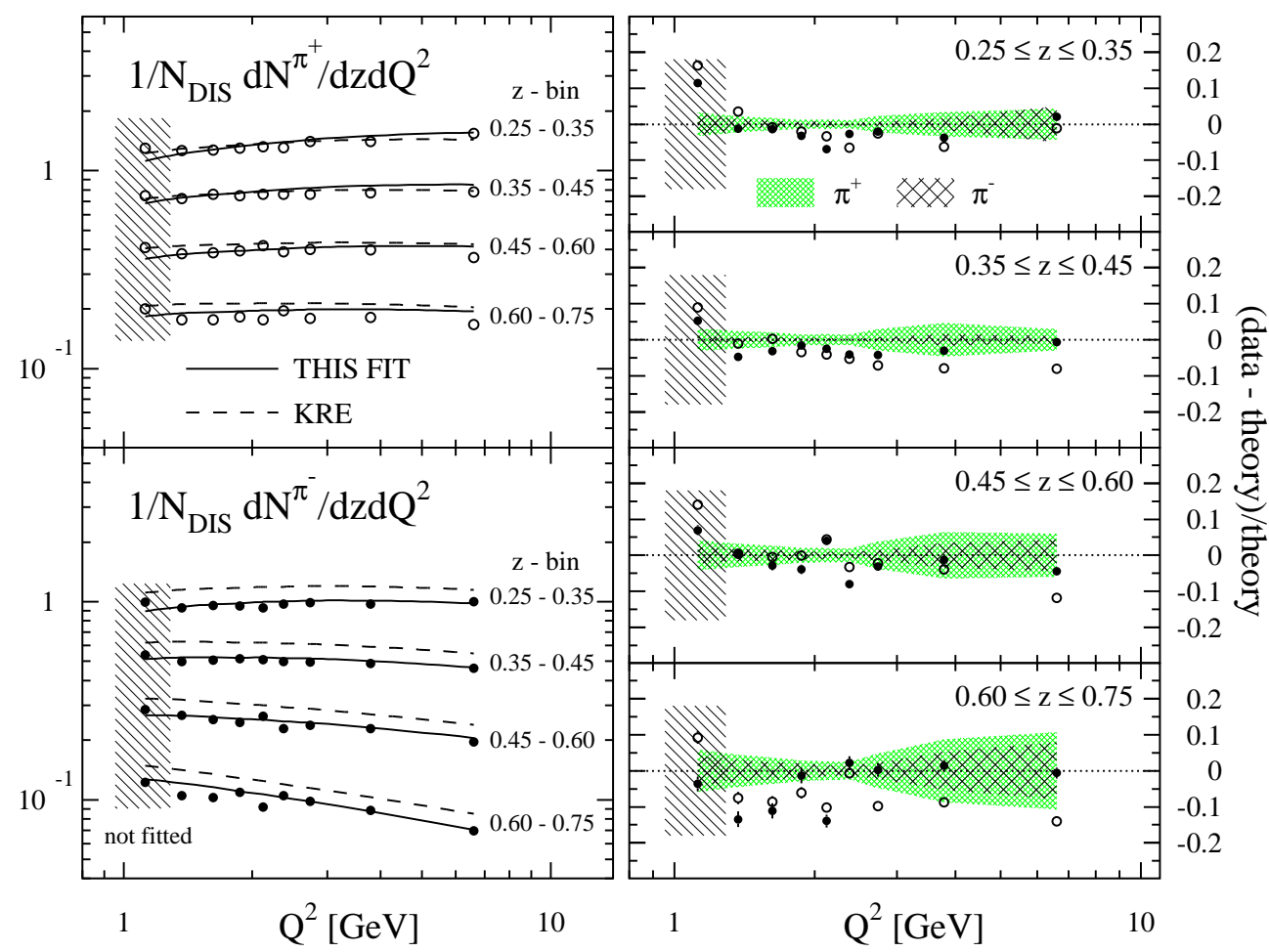

FIG. 4: L.h.s. comparison of our NLO results for charged pion multiplicities in SIDIS, $\left(1 / N_{D I S}\right) d N^{\pi^{ \pm}} / d z d Q^{2}$, with preliminary HERMES data [18]. Also shown are the results obtained with the KRE [7] parametrization. R.h.s. "(data-theory)/theory" for our NLO results, open and full circles denote $\pi^{+}$and $\pi^{-}$multiplicities, respectively. The shaded bands indicate estimates of theoretical uncertainties due to finite bin-size effects (see text). 


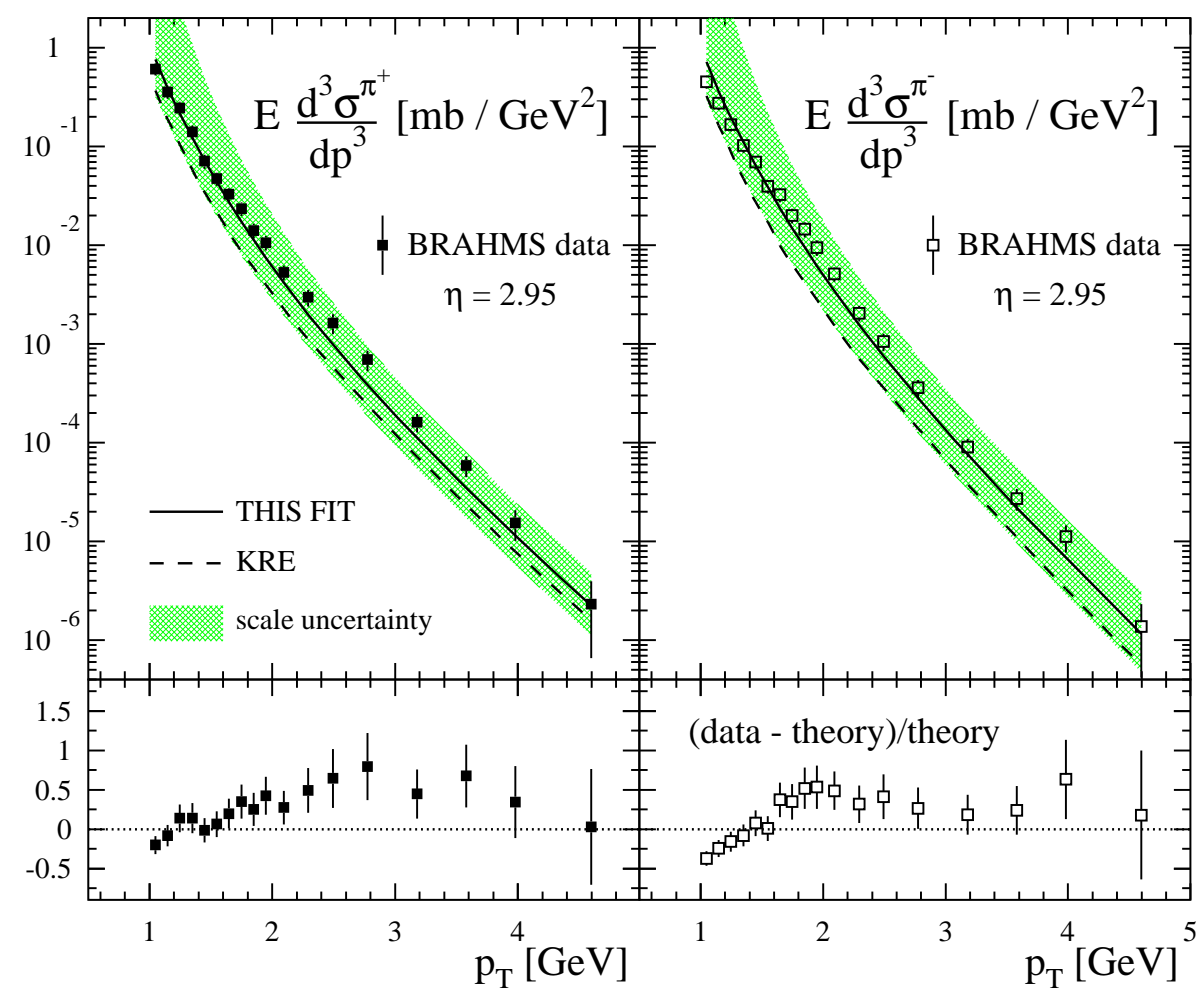

FIG. 5: upper panels comparison of our NLO results for single-inclusive charged pion production $p p \rightarrow \pi^{ \pm} X$ at rapidity $\eta=2.95$ (solid lines) with BRAHMS data [21] using $\mu_{f}=\mu_{r}=p_{T}$. Also shown are the results obtained with the KRE [7] parametrization (dashed lines). The shaded bands indicate theoretical uncertainties when all scales are varied in the range $p_{T} / 2 \leq \mu_{f}=\mu_{r} \leq 2 p_{T}$. lower panels "(data-theory)/theory" for our NLO results.

modern sets, e.g., [6].

The fit not only reproduces accurately the normalization in each $z$-bin for both $\pi^{+}$and $\pi^{-}$, but also the "scaling violations" in $Q^{2}$, which are rather large at the low scales involved in the experiment. Using the KRE fragmentation functions instead, also nicely reproduces the data in the $z$ bins up to 0.6 for the $\pi^{+}$multiplicities but considerably overshoots all $\pi^{-}$data, indicating that the assumed favored/unfavored separation [7] $D_{\bar{u}}^{\pi^{+}} / D_{u}^{\pi^{+}}=(1-z)$ is not accurate enough. We note that AKK [9] refrains from imposing any assumptions in their fit, which are beyond what can be obtained from SIA data. Hence, the AKK (and also KKP [8]) sets cannot be used in theoretical calculations whenever the experiment discriminates between positively or negatively charged pions (or kaons). This, of course, seriously restricts the potential applications and testability of these sets.

Again, as can be inferred from Tab. III the individual $\chi^{2}$ from SIDIS is fairly large, in particular for the $\pi^{-}$multiplicities. As in SIA, these data are based on a sample with high statistics and hence the accuracy of the data is very good. As before, any deviations between theory and data are severely punished by a large contribution to $\chi^{2}$. It is mainly the bin at the largest $z$ which makes all the trouble and contributes most to the total $\chi^{2}$. The optimum fit already "negotiates" the best compromise in describing these data. As mentioned in Sec. IIIB, we include an estimate of the theoretical uncertainties due to finite bin-size effects in the $\chi^{2}$ minimization. These are indicated by the shaded bands on the r.h.s. of Fig. 4. We also note, that we do not include the first data point in each $z$ bin in the fit as the scale $Q$ almost coincides with the already low input scale of our fit. In general, one may wonder about possible contaminations from, say, higher twists at the low $Q^{2}$ values accessible by HERMES. However, we do not find any indications that the SIDIS data are incompatible with the other data sets in our global analysis.

In Figs. 5- 7 we compare the results of our fit to recent data from proton-proton collisions at $\sqrt{s}=200 \mathrm{GeV}$ at RHIC. A general characteristics of all hadronic data is a large theoretical uncertainty associated with the choice of the arbitrary factorization and renormalization scales $\mu_{f}$ and $\mu_{r}$, respectively, in Eq. (14). Although largely reduced when going from the LO to the NLO approximation for the $p p \rightarrow \pi X$ cross section, as demonstrated in [25, 33], theoretical errors remain much more sizable than experimental errors. For our analysis we choose the transverse momentum of the produced pion as the hard scale, i.e., $\mu_{f}=\mu_{r}=p_{T}$, which yields a very good overall description at both central and forward rapidities. The shaded bands in Figs. 5 - 7 indicate theoretical uncertainties when all scales are varied simultaneously in the 


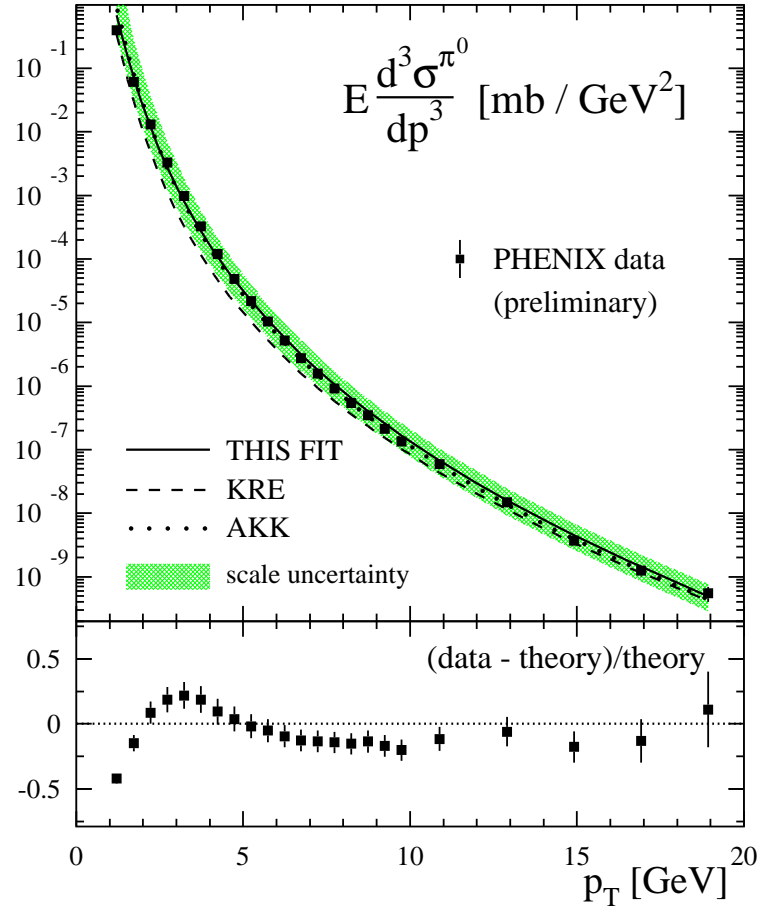

FIG. 6: Same as in Fig. 5 but now for single-inclusive neutral pion production $p p \rightarrow \pi^{0} X$ at mid-rapidities $|\eta| \leq 0.35$ measured by PHENIX [19].

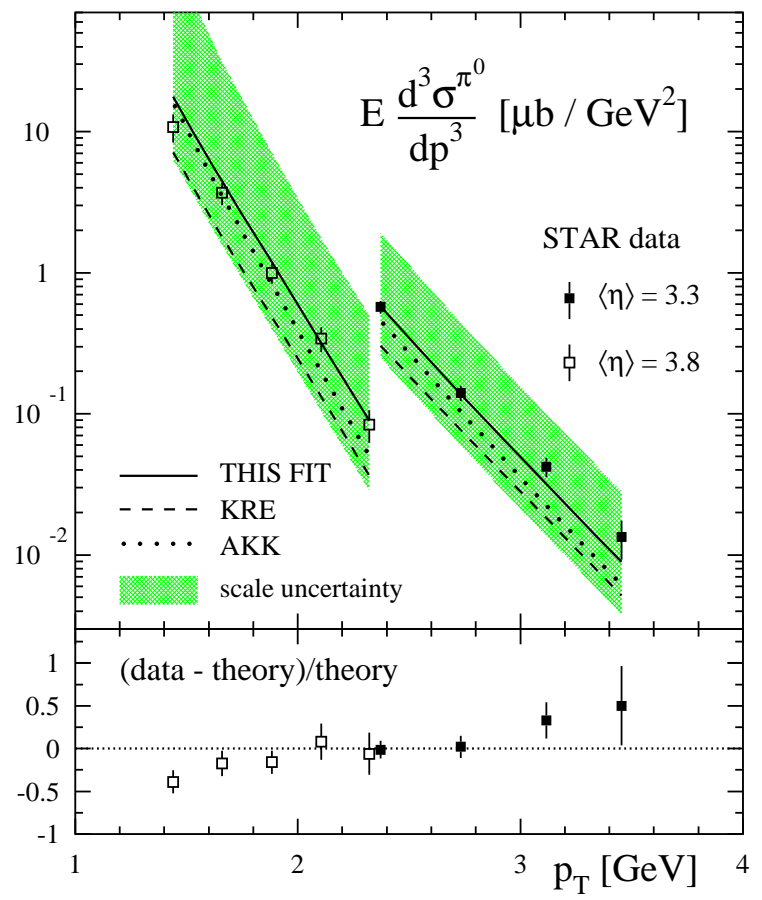

FIG. 7: Same as in Fig. 6 but now for STAR data 20] at forward rapidities $\langle\eta\rangle=3.3$ and $\langle\eta\rangle=3.8$, solid and open squares, respectively.

"usual" range $p_{T} / 2 \leq \mu_{f}=\mu_{r} \leq 2 p_{T}$.

In spite of the large uncertainties, the value of the

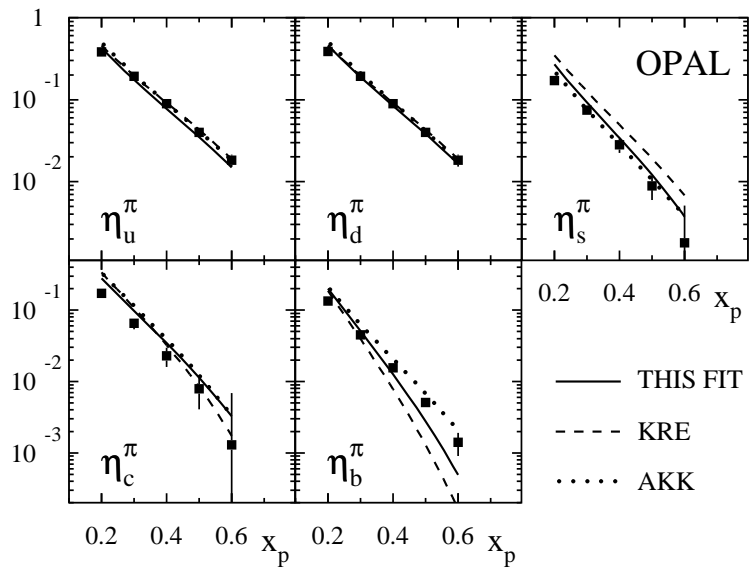

FIG. 8: Comparison of the OPAL "tagging probabilities" 14] for charged pions, $\eta_{i}^{\pi}$, as a function of the minimum $x_{p}$, see Eq. (22), with our NLO results (solid lines). Also shown are the results obtained with the KRE [7] and AKK [9] parameterizations, dashed and dotted lines, respectively.

RHIC data in the global analysis is manifold: data at central rapidities $\eta \simeq 0$ and not too large $p_{T}$ are strongly dominated by $g g \rightarrow g X$ scattering and hence constrain the gluon fragmentation $D_{g}^{\pi}$. At forward rapidities $\eta \simeq 3$ the mixture between quark and gluon fragmentation is roughly equal. It turns out that for both central and forward rapidities, the fragmentation occurs at fairly large average $\langle z\rangle \gtrsim 0.5$, see, e.g., Fig. 4 in Ref. [45], where information from SIA and SIDIS is sparse. As in case of SIDIS, the relevant hard scale of the process, $Q=\mathcal{O}\left(p_{T}\right)$, is much smaller than in SIA, thereby allowing to exploit evolution effects to further constrain the fragmentation functions.

The charged separated pion data obtained by BRAHMS very recently 21] and shown in Fig. 5 , nicely back up the separation of favored and unfavored fragmentation functions obtained from the SIDIS data discussed above. Another important feature of the BRAHMS and the RHIC data in general, is the failure of the KRE set to reproduce them. As can be seen in Figs. 5- 7 using the KRE fragmentation one considerably undershoots all RHIC data. Only by pushing the factorization scales to the extreme this could be remedied to some extent. This observation has usually been taken as an indication of an inadequately small gluon fragmentation function in the KRE set at intermediate-to-large $z$ and scales of a few $\mathrm{GeV}$. The fact that the agreement with the PHENIX data in Fig. 6 is much better at large $p_{T}$ when quark fragmentation becomes more important, supports this picture.

The recent AKK set 9] (as well as the preceding KKP analysis [8]) are characterized by a much larger gluon fragmentation function than in KRE and, consequently, leads to a good description of the PHENIX data and, to a lesser extent, also of the STAR data. The latter may suggest the need for an even larger gluon fragmentation function. As for SIDIS, the KKP or AKK sets cannot be used to compare to the charge separated BRAHMS 


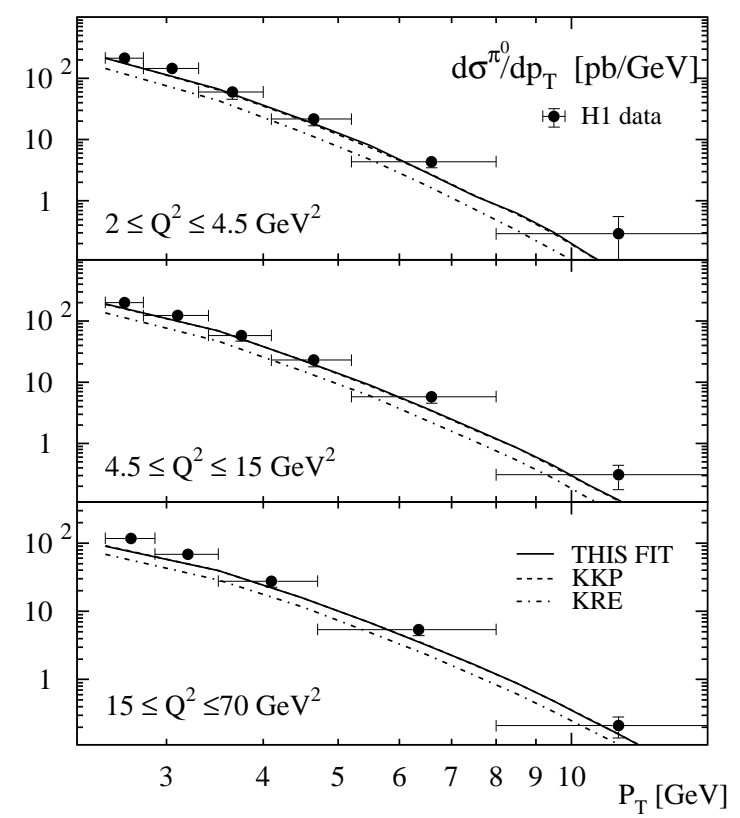

FIG. 9: Transverse momentum distributions for neutral pions obtained by $\mathrm{H} 1$ [46] in deep-inelastic $e^{+} p$ collisions compared to a NLO prediction using our new set of pion fragmentation functions. Also shown are the results obtained with the KRE [7] and KKP [8] parameterizations.

data. Since the KRE, KKP, and AKK sets are based on the analysis of roughly the same SIA data, the huge difference between the obtained gluon distributions only demonstrates again that a global analysis is imperative in obtaining reliable fragmentation functions. We will discuss the individual fragmentation functions and their uncertainties in some detail in Secs. IVE and IVF respectively. We already note here, that, surprisingly, the gluon fragmentation function obtained in our combined fit turns out to be smaller than in AKK at intermediate $z$, $0.2 \lesssim z \lesssim 0.5$, and only becomes larger at $z \gtrsim 0.6$. This is indicative of the complex interplay of the information provided by the different data sets in a global analysis.

The remaining data set used to constrain the pion fragmentation functions are the OPAL "tagging probabilities" [14] as defined in Eq. (22). As can be inferred from Fig. 8, all sets reproduce nicely the "data" for up and down flavors, which indicates that they are already essentially fixed by SIA data. For strangeness fragmentation, however, the sets differ considerably. In the KRE analysis [7], $D_{s}^{\pi}$ is completely fixed by assuming $N^{\prime}=1$ in Eq. (18), which overshoots significantly the OPAL result for $\eta_{s}^{\pi}$. For heavy flavors, in particular for charm, the agreement is less favorable for all sets. The results for $\eta_{c}^{\pi}$ from OPAL are clearly at odds with the tagged SIA data from TPC and SLD shown in Fig. 3. However, as explained in Sec. IIIB, the interpretation of the OPAL results beyond the LO should be taken with a grain of salt. So is not surprising to find some discrepancy in our fit where, contrary to AKK [9], the flavor separation comes not only from the OPAL results but also from the interplay of other data sets, which have a straight- forward and reliable interpretation in pQCD. The fairly large $\chi^{2}$ values we obtain for $\eta_{s}^{\pi}, \eta_{c}^{\pi}$, and $\eta_{b}^{\pi}$ are therefore not alarming and perhaps even expected. Nevertheless, it is encouraging that the general trend of the data is roughly reproduced by our combined global fit. We have also checked, that the outcome of our analysis does not change, if we excluded the OPAL results from out fit.

Our newly obtained NLO pion fragmentation functions are best tested in predictions for cross sections not included in the global analysis. As a final cross check we therefore compare in Fig. 9 measurements by the $\mathrm{H} 1 \mathrm{col}-$ laboration [46] of forward neutral pions in deep-inelastic positron-proton collisions at $\sqrt{s} \simeq 300 \mathrm{GeV}$ with $\mathrm{NLO}$ predictions based on our new set of fragmentation functions. The pions are required to be produced within a small angle $\theta_{\pi} \in\left[5^{\circ}, 25^{\circ}\right]$ from the direction of the proton beam in the laboratory frame, with an energy fraction $x_{\pi}=E_{\pi} / E_{P}>0.01$ and transverse momenta in the range $2.5<p_{T}<15 \mathrm{GeV}$. The observable has been shown to be crucially dependent on NLO contributions associated to the gluon fragmentation function [47]. The nice agreement between the data and the NLO results based on our new set of pion fragmentation functions is reassuring.

\section{B. LO analysis of pion fragmentation functions}

For completeness, we have also performed a global analysis of the same set of data given in Tab. II where all observables, $\alpha_{s}$, and the scale evolution of the fragmentation functions are computed at LO accuracy. We use the same parametrization (15) and fitting procedure as in the NLO case and outlined in Sec. III] The parameters of the optimum LO fit are given in Tab. III.

As it can be immediately seen from Tab. IV the quality of the LO fit is significantly worse than the NLO one, resulting in a $25 \%$ increase in the total $\chi^{2}$. Although all individual observables show an increase in $\chi^{2}$, it is somewhat more noticeable for proton-proton collision data. This is a sensible and expected result as the NLO corrections are known to be fairly large and important [25, 33]. To make up for the smaller LO partonic scattering cross

TABLE III: As in Tab. \but now describing the LO fragmentation functions for positively charged pions, $D_{i}^{\pi^{+}}\left(z, \mu_{0}\right)$.

\begin{tabular}{cccccc}
\hline \hline flavor $i$ & $N_{i}$ & $\alpha_{i}$ & $\beta_{i}$ & $\gamma_{i}$ & $\delta_{i}$ \\
\hline$u+\bar{u}$ & 0.367 & -0.228 & 1.20 & 5.29 & 4.51 \\
$d+\bar{d}$ & 0.404 & -0.228 & 1.20 & 5.29 & 4.51 \\
$\bar{u}=d$ & 0.117 & 0.123 & 2.19 & 7.80 & 6.80 \\
$s+\bar{s}$ & 0.197 & 0.123 & 2.19 & 7.80 & 6.80 \\
$c+\bar{c}$ & 0.256 & -0.310 & 4.89 & 0.00 & 0.00 \\
$b+\bar{b}$ & 0.469 & -1.108 & 6.45 & 0.00 & 0.00 \\
$g$ & 0.493 & 1.179 & 2.83 & -1.00 & 6.76 \\
\hline \hline
\end{tabular}


TABLE IV: Same as in Tab. II but now at LO accuracy.

\begin{tabular}{|c|c|c|c|c|}
\hline experiment & $\begin{array}{l}\text { data } \\
\text { type }\end{array}$ & $\begin{array}{l}\text { rel. norm. } \\
\text { in fit }\end{array}$ & $\begin{array}{c}\text { data points } \\
\text { fitted }\end{array}$ & $\chi^{2}$ \\
\hline \multirow[t]{4}{*}{$\mathrm{TPC}$ [15] } & incl. & 0.94 & 17 & 22.7 \\
\hline & "uds tag" & 0.94 & 9 & 1.9 \\
\hline & "c tag" & 0.94 & 9 & 5.6 \\
\hline & "b tag" & 0.94 & 9 & 7.3 \\
\hline \multirow[t]{2}{*}{ TASSO [17] } & incl. $(34 \mathrm{GeV})$ & 0.94 & 11 & 48.1 \\
\hline & incl. $(44 \mathrm{GeV})$ & 0.94 & 7 & 21.5 \\
\hline \multirow[t]{4}{*}{ SLD [16] } & incl. & 1.007 & 28 & 20.9 \\
\hline & "uds tag" & 1.007 & 17 & 21.3 \\
\hline & "c tag" & 1.007 & 17 & 9.3 \\
\hline & "b tag" & 1.007 & 17 & 34.5 \\
\hline ALEPH [11] & incl. & 0.97 & 22 & 64.4 \\
\hline \multirow[t]{3}{*}{ DELPHI [12] } & incl. & 1.0 & 17 & 45.9 \\
\hline & "uds tag" & 1.0 & 17 & 30.6 \\
\hline & "b tag" & 1.0 & 17 & 51.9 \\
\hline \multirow[t]{6}{*}{ OPAL $[13,14]$} & incl. & 1.0 & 21 & 20.7 \\
\hline & $" u$ tag" & 1.10 & 5 & 9.3 \\
\hline & "d tag" & 1.10 & 5 & 7.5 \\
\hline & "s tag" & 1.10 & 5 & 66.7 \\
\hline & "c tag" & 1.10 & 5 & 36.9 \\
\hline & "b tag" & 1.10 & 5 & 88.8 \\
\hline \multirow[t]{2}{*}{ HERMES [18] } & $\pi^{+}$ & 1.03 & 32 & 53.6 \\
\hline & $\pi^{-}$ & 1.03 & 32 & 153.9 \\
\hline PHENIX [19] & $\pi^{0}$ & 1.09 & 23 & 82.2 \\
\hline \multirow[t]{2}{*}{ STAR [22] } & $\pi^{0},\langle\eta\rangle=3.3$ & 0.95 & 4 & 15.5 \\
\hline & $\pi^{0},\langle\eta\rangle=3.7$ & 0.95 & 5 & 11.7 \\
\hline \multirow[t]{2}{*}{ BRAHMS [21] } & $\pi^{+},\langle\eta\rangle=2.95$ & 1.0 & 18 & 46.3 \\
\hline & $\pi^{-},\langle\eta\rangle=2.95$ & 1.0 & 18 & 77.7 \\
\hline TOTAL: & & & 392 & 1056.8 \\
\hline
\end{tabular}

sections relevant for RHIC data, the most striking difference between the LO and NLO fragmentation functions is found for gluons, while the moments for the quark flavors remain rather stable; cf. Tabs. [I and III In spite of the larger $\chi^{2}$, for consistency, our LO sets should be used for rough estimates of observables where NLO corrections are not yet available, or in event generators based on matrix elements at LO accuracy. Because of the limited usefulness of the LO set, we refrain from going into any further details here.

\section{NLO analysis of kaon fragmentation functions}

Our NLO fits to single inclusive kaon production data as compared to those for pions, reflect the sensible difference in quality of both data sets. Even the most precise kaon production data in SIA exhibit experimental uncertainties typically twice as large as those found for pions. The potentially problematic low- $z$ region is ex-
TABLE V: Data used in the NLO global analysis of kaon fragmentation functions, the individual $\chi^{2}$ values for each set, the fitted normalizations, and the total $\chi^{2}$ of the fit.

\begin{tabular}{|c|c|c|c|c|}
\hline experiment & $\begin{array}{l}\text { data } \\
\text { type }\end{array}$ & $\begin{array}{c}\text { rel. norm. } \\
\text { in fit }\end{array}$ & $\begin{array}{c}\text { data points } \\
\text { fitted }\end{array}$ & $\chi^{2}$ \\
\hline $\mathrm{TPC}$ [15] & incl. & 0.94 & 12 & 9.5 \\
\hline \multirow[t]{4}{*}{ SLD [16] } & incl. & 0.983 & 18 & 14.4 \\
\hline & "uds tag" & 0.983 & 10 & 14.4 \\
\hline & "c tag" & 0.983 & 10 & 17.2 \\
\hline & "b tag" & 0.983 & 10 & 15.2 \\
\hline ALEPH [11] & incl. & 0.97 & 13 & 12.3 \\
\hline \multirow[t]{3}{*}{ DELPHI [12] } & incl. & 1.0 & 12 & 1.0 \\
\hline & "uds tag" & 1.0 & 12 & 2.3 \\
\hline & "b tag" & 1.0 & 12 & 4.3 \\
\hline \multirow[t]{5}{*}{ OPAL [14] } & " $u$ tag" & 1.10 & 5 & 6.5 \\
\hline & "d tag" & 1.10 & 5 & 9.9 \\
\hline & "s tag" & 1.10 & 5 & 36.8 \\
\hline & "c tag" & 1.10 & 5 & 44.9 \\
\hline & "b tag" & 1.10 & 5 & 18.6 \\
\hline \multirow[t]{2}{*}{ HERMES [18] } & $K^{+}$ & 1.03 & 24 & 15.0 \\
\hline & $K^{-}$ & 1.03 & 24 & 79.3 \\
\hline STAR [20] & $K_{S}^{0}$ & 0.95 & 14 & 40.0 \\
\hline \multirow[t]{2}{*}{ BRAHMS [21] } & $K^{+},\langle\eta\rangle=2.95$ & 1.0 & 18 & 28.8 \\
\hline & $K^{-},\langle\eta\rangle=2.95$ & 1.0 & 18 & 21.5 \\
\hline TOTAL: & & & 232 & 394.1 \\
\hline
\end{tabular}

TABLE VI: Parameters describing the NLO fragmentation functions for positively charged kaons, $D_{i}^{K^{+}}\left(z, \mu_{0}\right)$, at the input scale $\mu_{0}=1 \mathrm{GeV}$. Inputs for the charm and bottom fragmentation functions refer to $\mu_{0}=m_{c}=1.43 \mathrm{GeV}$ and $\mu_{0}=m_{b}=4.3 \mathrm{GeV}$, respectively.

\begin{tabular}{cccccc}
\hline \hline flavor $i$ & $N_{i}$ & $\alpha_{i}$ & $\beta_{i}$ & $\gamma_{i}$ & $\delta_{i}$ \\
\hline$u+\bar{u}$ & 0.058 & 0.705 & 1.20 & 15.00 & 6.02 \\
$s+\bar{s}$ & 0.343 & -0.065 & 1.20 & 4.36 & 3.73 \\
$d+\bar{d}$ & 0.016 & 1.108 & 10.00 & 10.00 & 3.28 \\
$\bar{u}=s$ & 0.008 & 1.108 & 10.00 & 10.00 & 3.28 \\
$c+\bar{c}$ & 0.196 & 0.102 & 4.56 & 0.00 & 0.00 \\
$b+\bar{b}$ & 0.139 & -0.584 & 7.42 & 0.00 & 0.00 \\
$g$ & 0.017 & 5.055 & 1.20 & 0.00 & 0.00 \\
\hline \hline
\end{tabular}

pected to set in earlier due to the larger kaon mass. To be on the safe side, we raise the cut from $z_{\text {min }}=0.05$ to $z_{\min }=0.1$. Clearly, one must expect much less well constrained fragmentation functions for kaons. Otherwise the $\chi^{2}$ minimization proceeds along the same lines as for the analysis of the pion data, and the results are summarized in Tab. V]. As demonstrated in Figs. 10-16, the overall agreement of our NLO fit and data is again remarkably good. The set of parameters specifying the obtained kaon fragmentation functions at NLO accuracy 

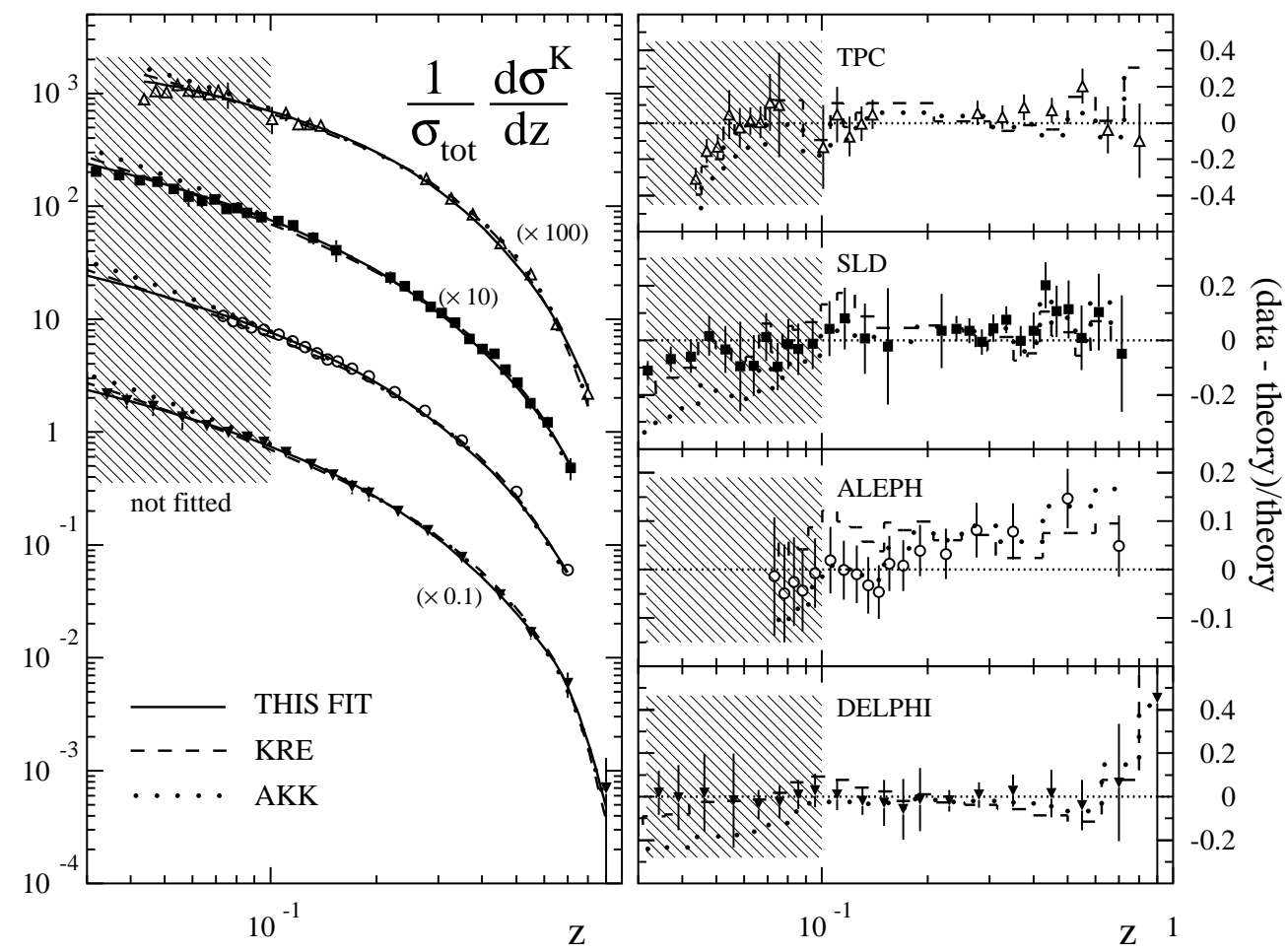

FIG. 10: As in Fig. 1 but now for inclusive kaon production in SIA.

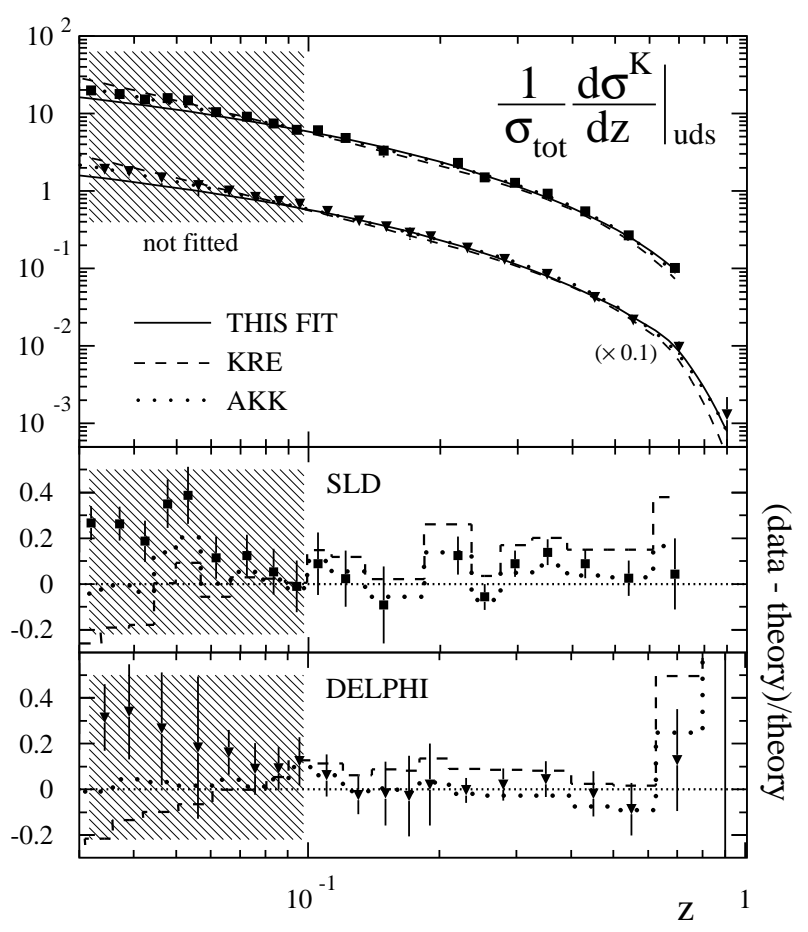

FIG. 11: Same as in Fig. 10 but now for light quark ("uds") tagged cross sections. The upper panel shows the comparison of the "uds" tagged cross sections with data and the lower two panels show "(data-theory)/theory".

can be found in Tab. VI.
Starting with the SIA data in Figs. 10 - 12, our fit shows again, thanks to the more flexible $z$-dependence of the initial distributions (15) and the less stringent flavor symmetry assumptions, a slightly better agreement with SIA data than previous analyses [7, 9]. Also, below $z_{\min }=0.1$ the fit still follows the trend of the data, which ensures that finite mass effects or the unstable small- $z$ behavior of the scale evolution are still negligible. In terms of $\chi^{2}$, none of the SIA data sets, even the ones for heavy flavor tagged cross sections in Fig. 12, poses any problems. The reduced statistical accuracy of the kaon data compared to the pion data is immediately obvious and also reflects itself in larger fluctuations in the "(data-theory)/theory" comparisons in Figs. 10- 12.

The biggest asset of our global analysis is again that not only SIA data but, for the first time, also SIDIS and RHIC data are nicely reproduced as is demonstrated in Figs. 13 - 15. It is worth noticing that, at variance with what happens for pions, the HERMES SIDIS data, Fig.13, rule out completely the flavor separation assumed in Ref. 7] for kaon fragmentation functions. The prediction based on the KRE set overshoots the data by a factor of two, whereas our global fit shows much better agreement. This is in particular true for the $K^{+}$multiplicities, which in SIDIS receive the dominant contribution related to the (large) up quark parton density in the proton. The production of $K^{-}$is linked predominantly to strange quarks and anti-up quarks in the proton, both of which are much less abundant than up quarks for the relatively large momentum fractions $x$ relevant for HER- 

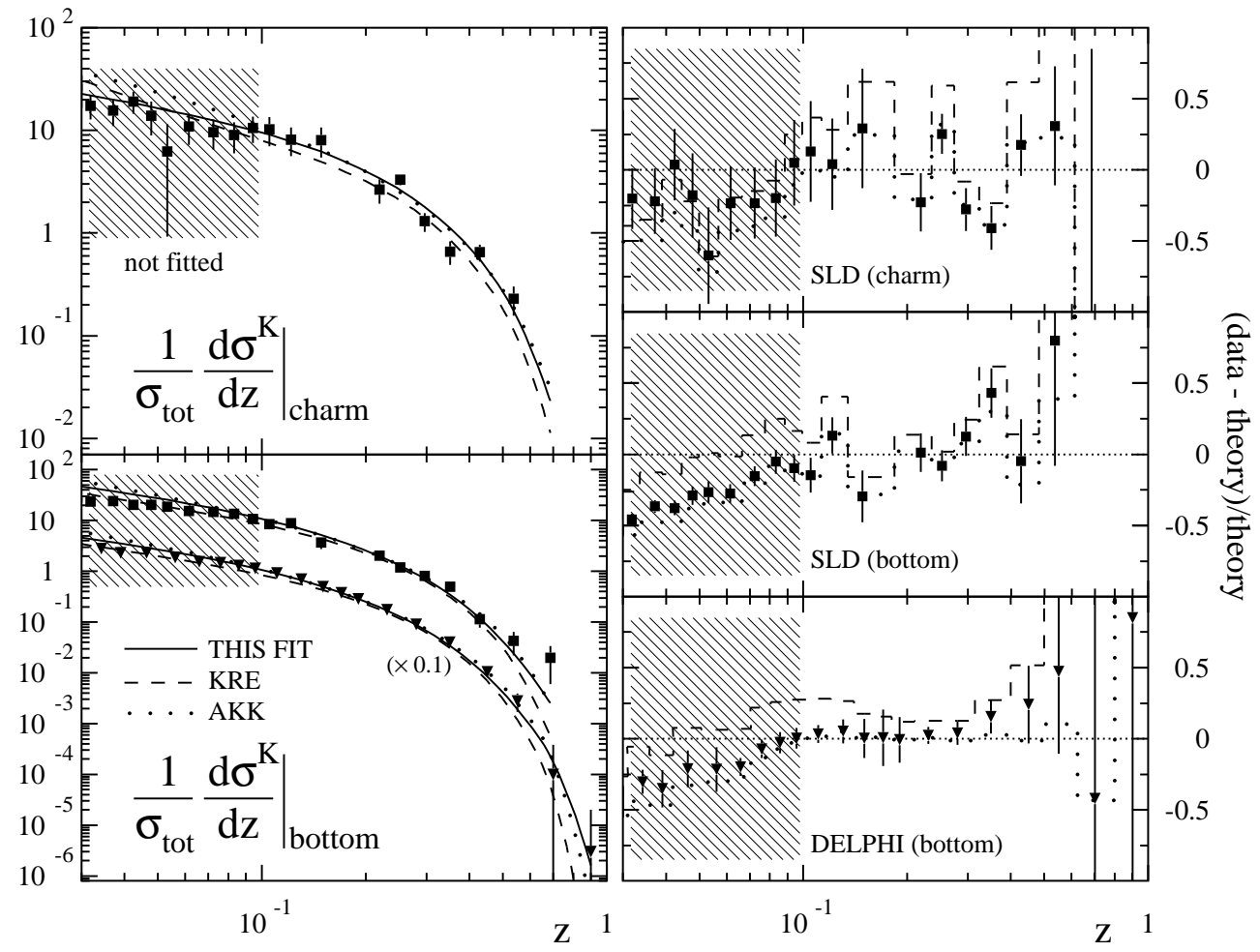

FIG. 12: Same as in Fig. 10 but now for charm and bottom quark tagged cross sections.

MES. Here our fit reproduces the magnitude of the $K^{-}$ multiplicities in each $z$ bin, but not the $Q^{2}$ slope. In fact, the related $\chi^{2}$ is the by far largest contribution to the total $\chi^{2}$ of the fit, cf. Tab. V The theoretical uncertainty introduced by the size of the bins in the computation of the cross section is also significantly larger than in the case of pions. For negatively charged kaons it is further amplified by the rapid growth of sea quark densities in the proton with $Q^{2}$. Taking also into account that the strange and, to some extent, also the anti-up quark parton densities are not too well constraint, the agreement of the new fit is rather encouraging.

Our new set of kaon fragmentation functions also yields the best description of the BRAHMS [21] and STAR data [22], Figs. 14]and 15] respectively, which both probe the large $z$ regime, $z \gtrsim 0.5$, poorly mapped out by SIA data. As for pions, the KRE set undershoots all RHIC kaon data, whereas AKK overshoots the STAR $K_{S}^{0}$ data. As before, the AKK (and KKP) set does not allow to compute the charge separated $K^{+}$and $K^{-}$yields by BRAHMS. Again, the discrepancy with the RHIC data can be traced back to the behavior of the gluon fragmentation function at large $z$ and will be discussed further in Sec. IVE. Notice again the very large theoretical scale uncertainties for the RHIC data.

The last data set entering the global analysis of kaon fragmentation functions are the OPAL tagging probabilities $\eta_{i}^{K}$ [14]. In view of the already discussed conceptual problems with these data, the overall agreement with the OPAL results in Fig. 16 is reasonable. The lack of agree- ment, most noticeable for charm, again suggests some degree of inconsistency with other data sets included in the fit.

\section{LO analysis of kaon fragmentation functions}

The LO global analysis of kaon production data yields significantly larger values of $\chi^{2}$. The parameters describing the optimum LO kaon fragmentation functions are given in Tab. VII while $\chi^{2}$ contributions are in Tab. VIII,

The overall increase with respect to the NLO fit is about 30\%, even larger than the one found in the case of pions, and with the most noticeable differences in the partial contributions stemming from RHIC and SIDIS data.

TABLE VII: As in Tab. VI but now for the LO fragmentation functions for positively charged kaons.

\begin{tabular}{cccccc}
\hline \hline flavor $i$ & $N_{i}$ & $\alpha_{i}$ & $\beta_{i}$ & $\gamma_{i}$ & $\delta_{i}$ \\
\hline$u+\bar{u}$ & 0.054 & 1.018 & 1.20 & 15.00 & 6.04 \\
$s+\bar{s}$ & 0.361 & 0.733 & 1.20 & 20.00 & 5.28 \\
$\bar{u}=s$ & 0.005 & 1.322 & 10.00 & 10.00 & 3.67 \\
$d+\bar{d}$ & 0.010 & 1.322 & 10.00 & 10.00 & 3.67 \\
$c+\bar{c}$ & 0.214 & 0.239 & 4.27 & 0.00 & 0.00 \\
$b+\bar{b}$ & 0.147 & -0.464 & 7.37 & 0.00 & 0.00 \\
$g$ & 0.036 & 5.282 & 1.20 & 0.00 & 0.00 \\
\hline \hline
\end{tabular}



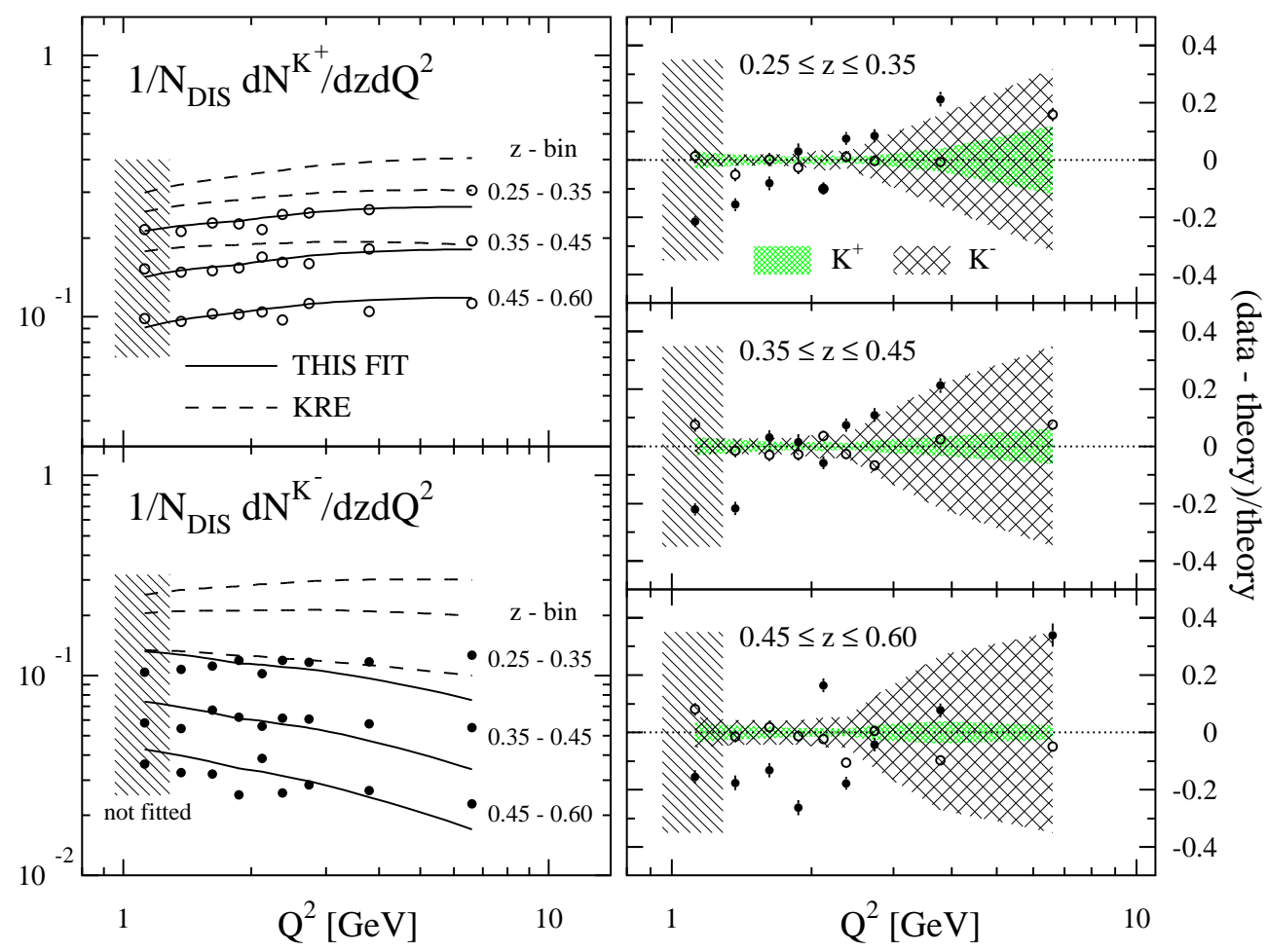

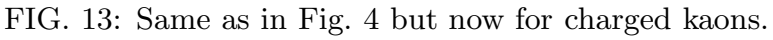

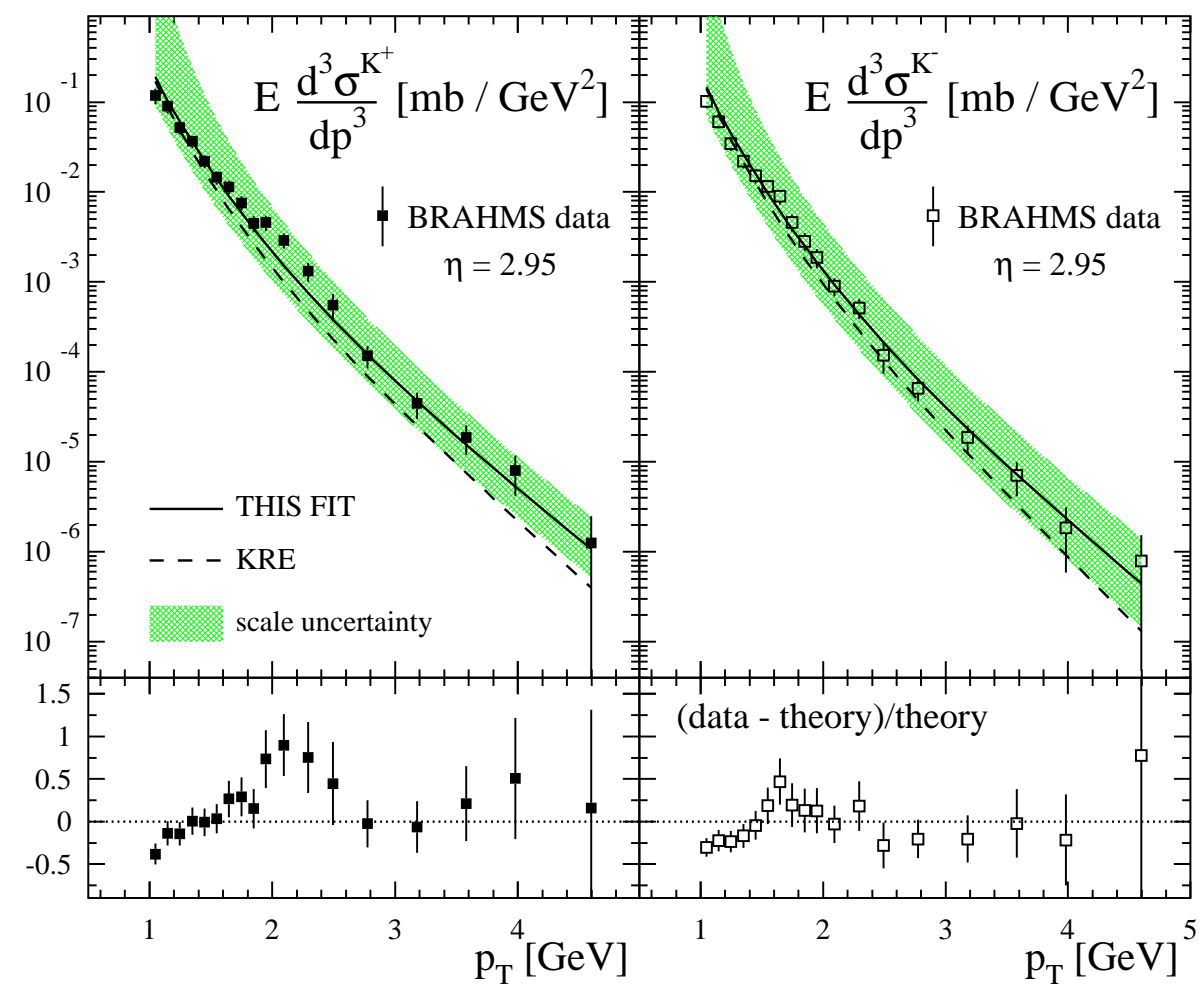

FIG. 14: Same as in Fig. 5 but now for charged kaons. 


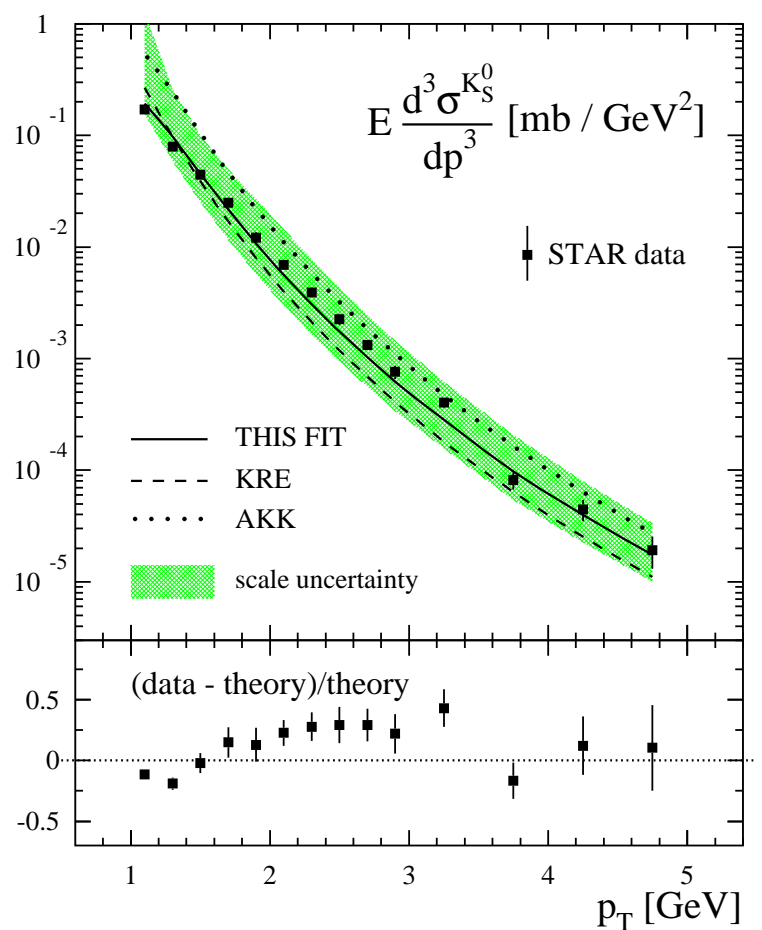

FIG. 15: Same as in Fig. 6 but now for STAR data 22] for $K_{S}^{0}$ production at central rapidities $|\eta| \leq 0.5$.

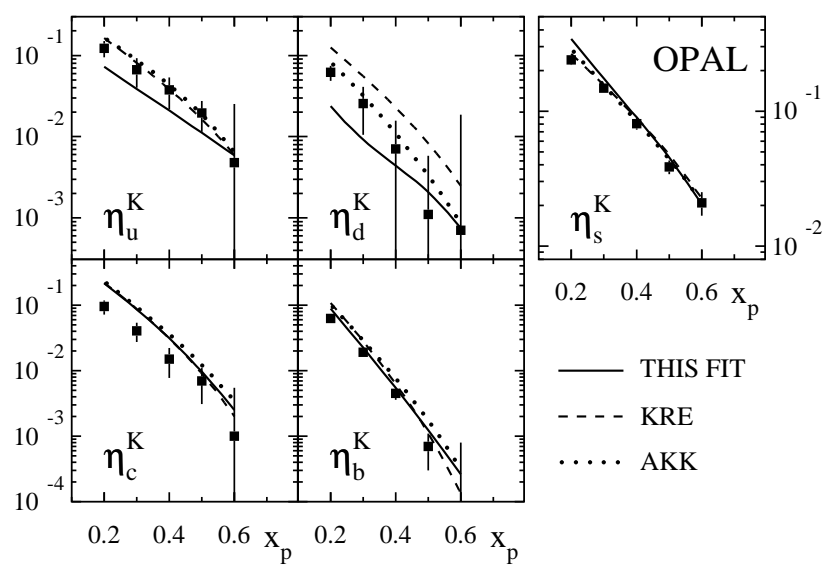

FIG. 16: Same as in Fig. 8 but now for charged kaons.

\section{E. Fragmentation Functions}

In this Section we shall present an overall description of the different fragmentation functions $D_{i}^{\pi^{+}, K^{+}}$obtained in the global fit [48] and perform a comparison with the KRE 7] and AKK 9] NLO sets based only on SIA data.

The upper panels of Fig. 17 show the fragmentation functions for positively charged pions at the scale $Q^{2}=10 \mathrm{GeV}^{2}$. As expected, the sum $u+\bar{u}$ dominates over the unfavored distributions $\bar{u}$ and $s$. At large values of $z$, there is an important contribution from the valence $u$ fragmentation, while at small $z$ the sea distribution dominates and $u+\bar{u} \simeq 2 \bar{u}$. In the same limit, it can be observed that the $s$ fragmentation function turns out
TABLE VIII: Same as in Tab. $\mathrm{V}$ but now at LO accuracy.

\begin{tabular}{|c|c|c|c|c|}
\hline experiment & $\begin{array}{l}\text { data } \\
\text { type }\end{array}$ & $\begin{array}{l}\text { rel. norm. } \\
\text { in fit }\end{array}$ & $\begin{array}{c}\text { data points } \\
\text { fitted }\end{array}$ & $\chi^{2}$ \\
\hline TPC [15] & incl. & 0.94 & 12 & 12.7 \\
\hline TASSO [17] & incl. $(34 \mathrm{GeV})$ & 0.94 & 4 & 2.6 \\
\hline \multirow[t]{4}{*}{ SLD [16] } & incl. & 0.983 & 18 & 18.1 \\
\hline & "uds tag" & 0.983 & 10 & 21.9 \\
\hline & "c tag" & 0.983 & 10 & 18.4 \\
\hline & "b tag" & 0.983 & 10 & 15.0 \\
\hline ALEPH [11] & incl. & 0.97 & 13 & 14.0 \\
\hline \multirow[t]{3}{*}{ DELPHI [12] } & incl. & 1.0 & 12 & 1.2 \\
\hline & "uds tag" & 1.0 & 12 & 2.6 \\
\hline & "b tag" & 1.0 & 12 & 4.1 \\
\hline \multirow[t]{5}{*}{ OPAL [14] } & " $u$ tag" & 1.10 & 5 & 7.8 \\
\hline & "d tag" & 1.10 & 5 & 10.6 \\
\hline & "s tag" & 1.10 & 5 & 32.9 \\
\hline & "c tag" & 1.10 & 5 & 53.1 \\
\hline & "b tag" & 1.10 & 5 & 19.7 \\
\hline \multirow[t]{2}{*}{ HERMES [18] } & $K^{+}$ & 1.03 & 24 & 23.9 \\
\hline & $K^{-}$ & 1.03 & 24 & 131.2 \\
\hline STAR [20] & $K_{S}^{0}$ & 0.95 & 14 & 59.0 \\
\hline \multirow[t]{2}{*}{ BRAHMS [21] } & $K^{+},\langle\eta\rangle=2.95$ & 1.0 & 18 & 36.9 \\
\hline & $K^{-},\langle\eta\rangle=2.95$ & 1.0 & 18 & 34.7 \\
\hline TOTAL: & & & 236 & 520.7 \\
\hline
\end{tabular}

to be smaller than the corresponding $\bar{u}$ sea distribution, as anticipated in Sec. IVA when discussing the value of the relevant parameter $N^{\prime}$. As can also be noticed from Fig. 17, charm and gluon fragmentation are quite sizable and comparable to the one of the light quarks at small $z$. This is actually a general feature of heavy quark fragmentation, opposite to the behavior of the usually less relevant heavy quark parton distributions. At this scale, the bottom channel has not opened yet, but the corresponding distribution can be observed in Fig. 18, where the same functions are plotted at a higher scale $Q^{2}=M_{Z}^{2}$. As expected, heavy quark and gluon fragmentation are rather suppressed at larger values of $z$.

In the middle and lower panels of Figs. 17 and [18, we compare our set of fragmentation functions to those from KRE and AKK, respectively. The largest differences appear for the unfavored quark and gluon distributions and, usually, both at large $z$ and near $z_{\text {min }}$ below which fragmentation functions cannot be used. Notice that for AKK, $D_{\bar{u}}^{\pi^{+}}$is not available for comparison and that their analysis is limited to $z>0.1$ rather than $z>z_{\min }=0.05$. Since AKK tends to overestimate the SIA cross-section outside the fitted region, i.e., below $z=0.1$, any disagreement there is not surprising.

While the discrepancy with KRE for the light quark distributions are reasonably moderate, there happens to be a rather large difference at the level of the strange fragmentation with AKK. The origin of this disagreement 


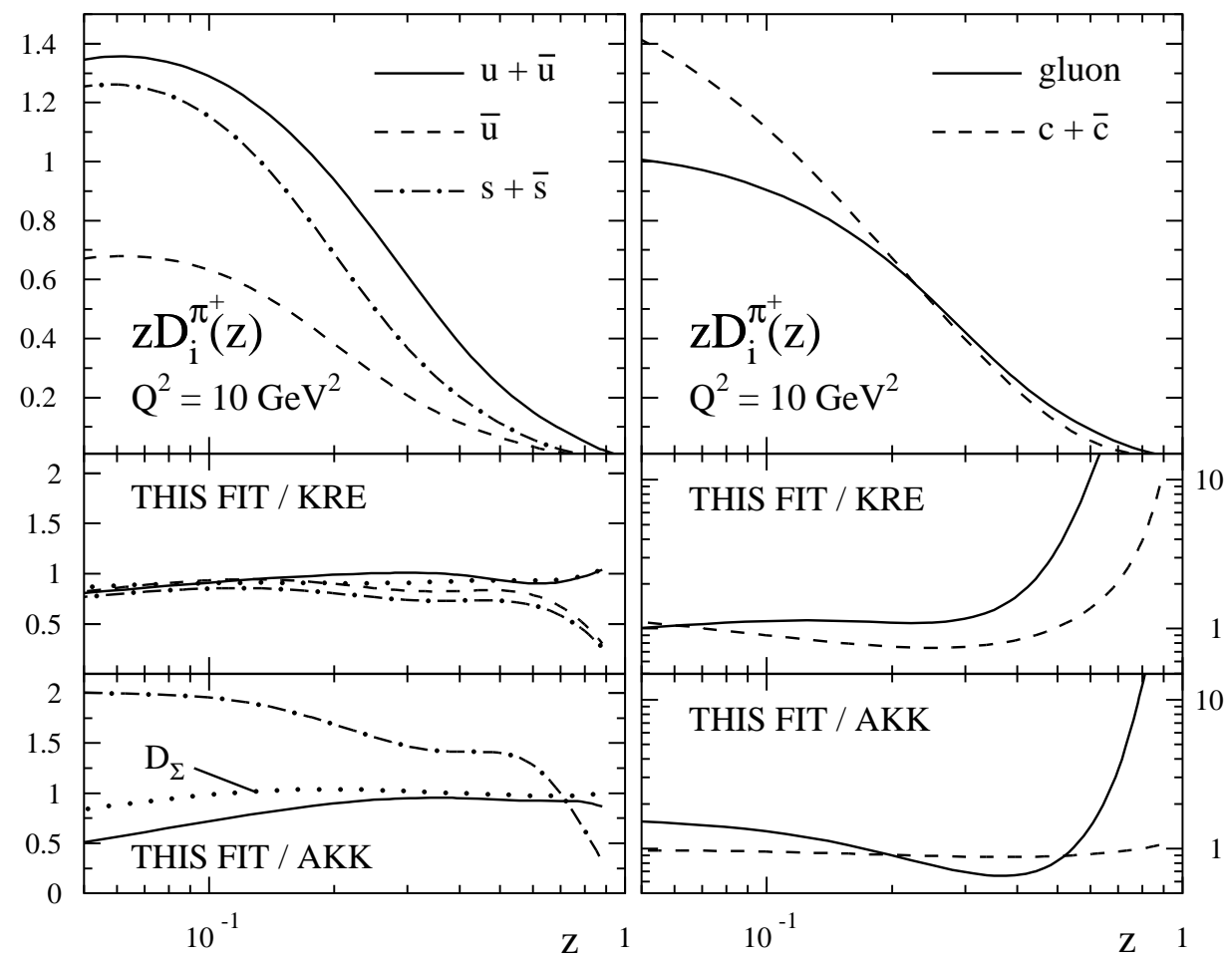

FIG. 17: upper panels individual fragmentation functions for positively charged pions $z D_{i}^{\pi^{+}}\left(z, Q^{2}\right)$ at $Q^{2}=10 \mathrm{GeV}^{2}$ for $i=u+\bar{u}, \bar{u}, s+\bar{s}, g$, and $c+\bar{c}$. middle panels ratios of our fragmentation functions to the ones of KRE [7]. The dotted line indicates the ratio for singlet combination of fragmentation functions $z D_{\Sigma}^{\pi^{+}}$. lower panels ratios of our fragmentation functions to the ones of AKK [9]; note that $D_{\bar{u}}^{\pi^{+}}$is not available in the AKK analysis.

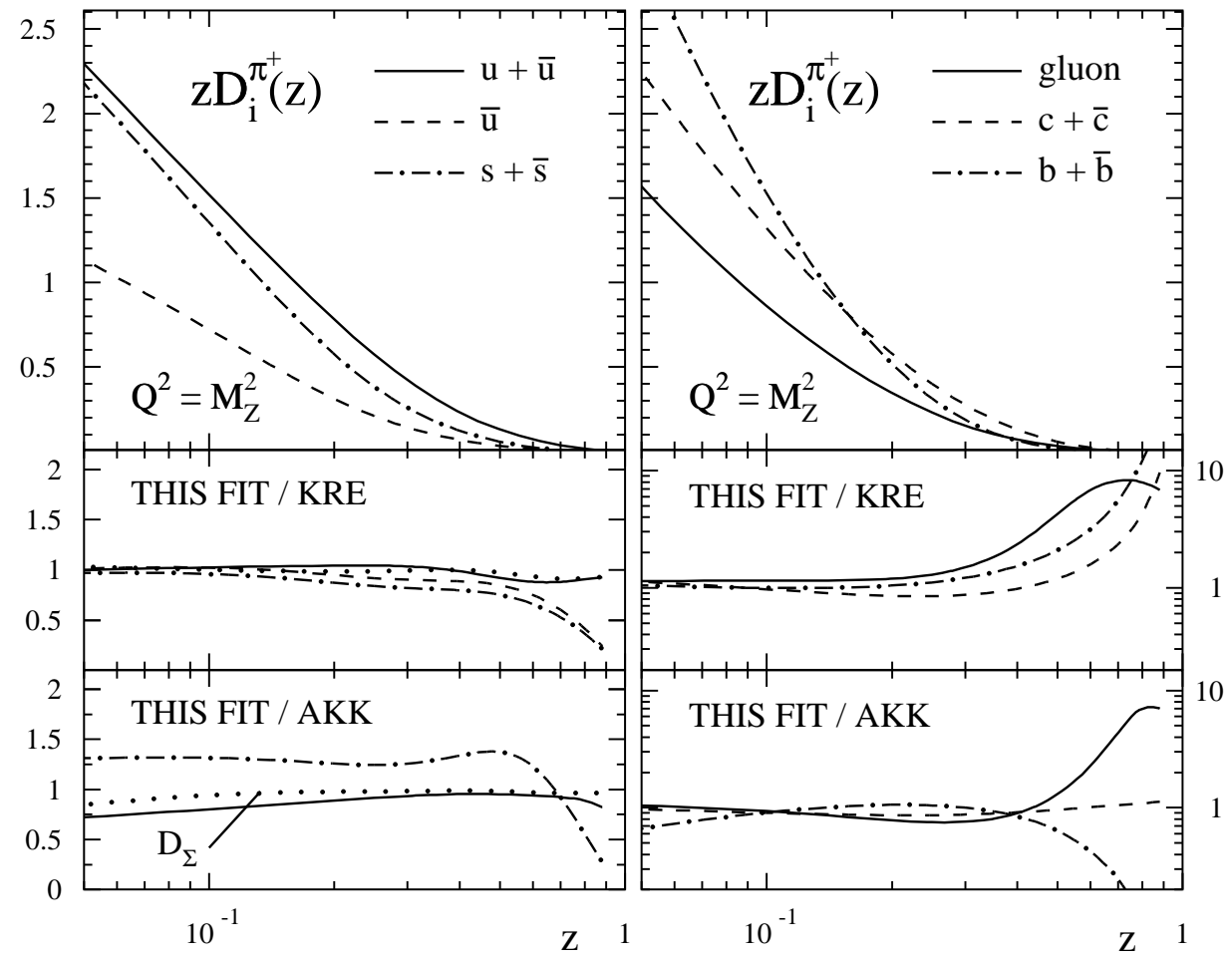

FIG. 18: Same as in Fig. 17 but now for $Q=M_{Z}$. Also shown is the $b+\bar{b}$ fragmentation function. 


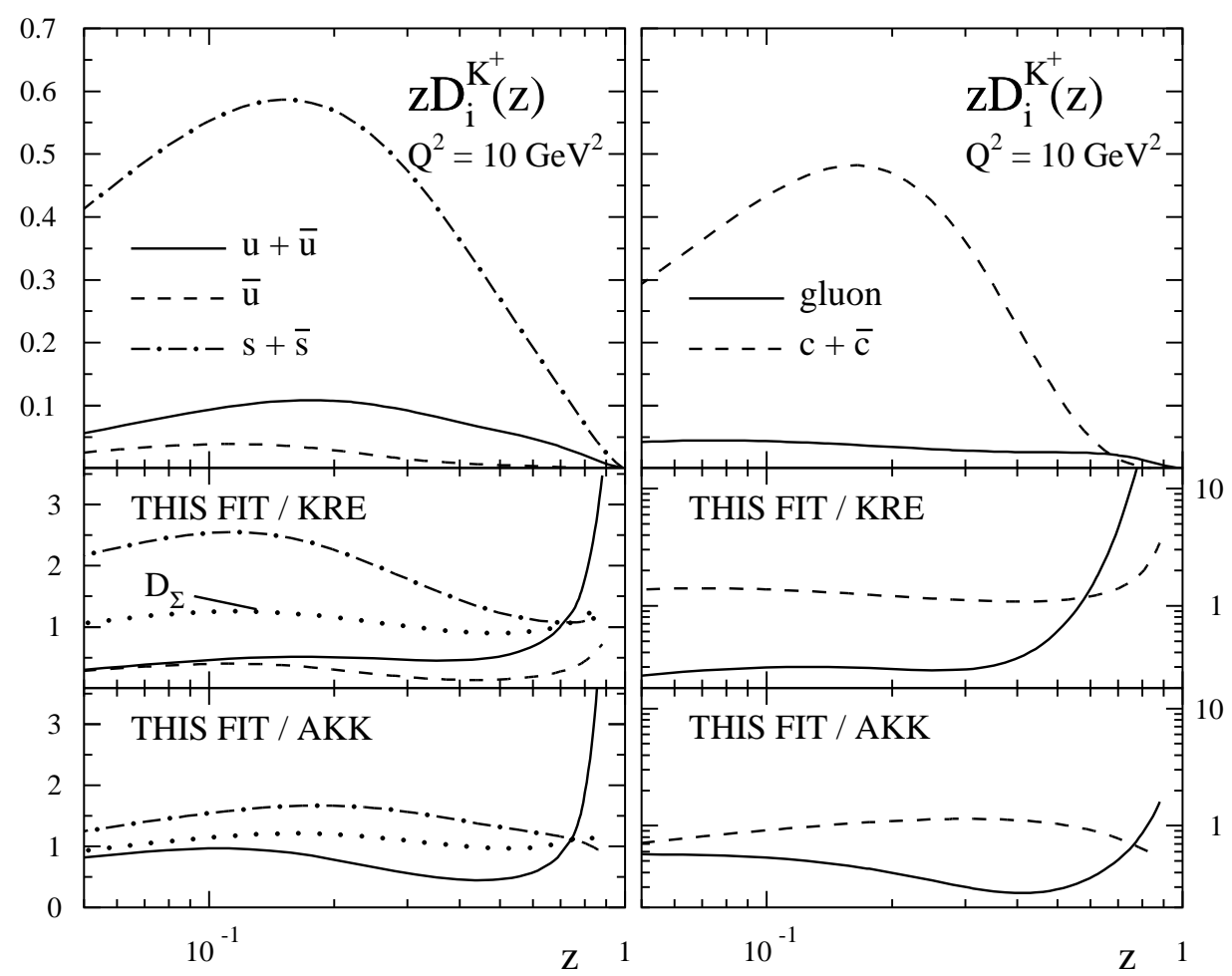

FIG. 19: Same as in Fig. 17 but now for positively charged kaons $K^{+}$.

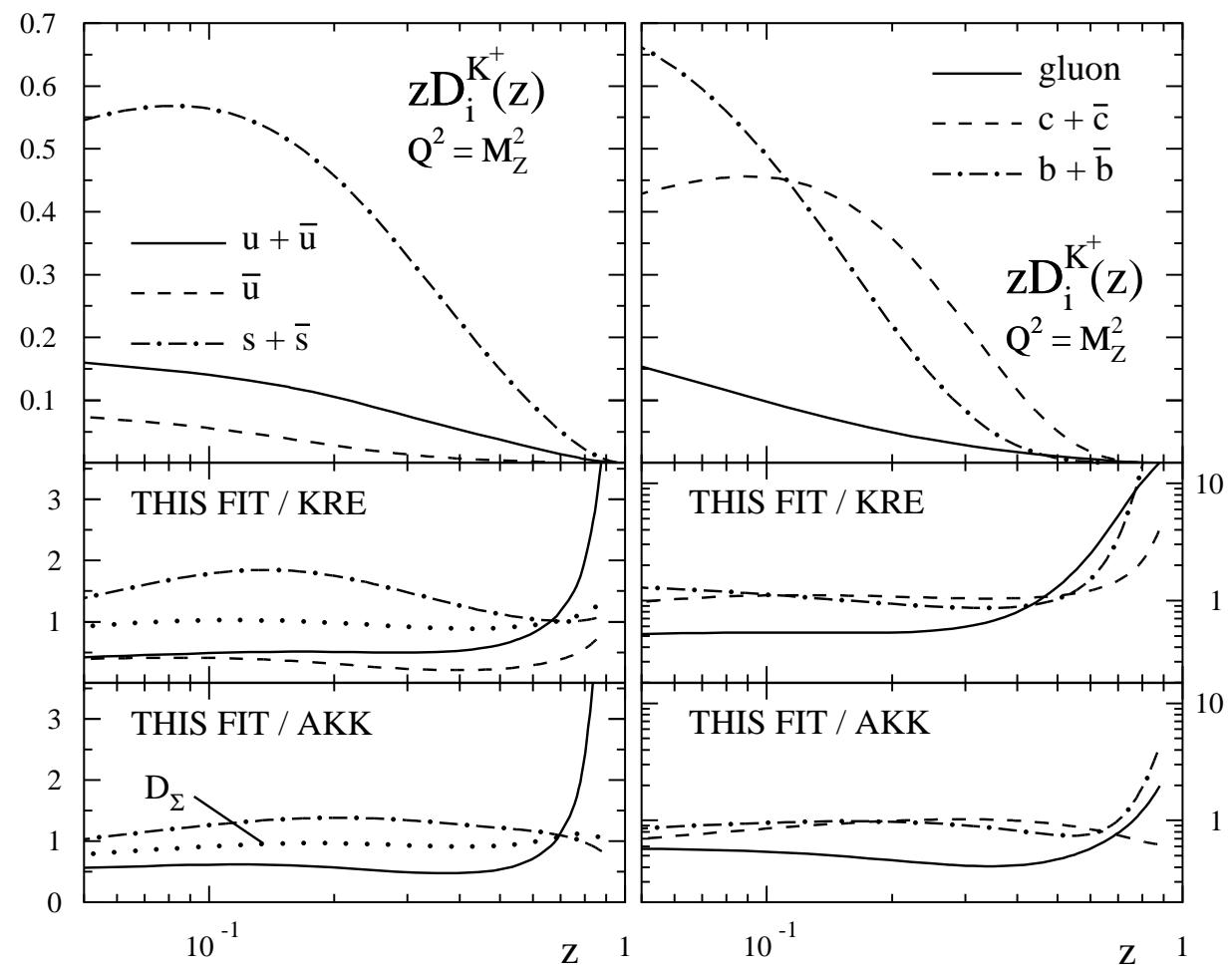

FIG. 20: Same as in Fig. 18 but now for positively charged kaons $K^{+}$. 
can be easily understood: SIDIS data, not included in the AKK fit, require a smaller fragmentation for $u$ (as can be seen in the same plot) and $d$ quarks. Since the singlet combination, $D_{\Sigma}$, is already well constraint by SIA data, as can also be seen in Figs. 17] and 18, that automatically requires an increase in the $D_{s}^{\pi^{+}}$fragmentation function.

The most pronounced differences appear at very large values of $z$ for both heavy flavor and gluon fragmentation functions. In the heavy quark case, both SIDIS and RHIC data are insensitive and SIA data suffer from very large experimental uncertainties for $z>0.4$ and even conflicting results. Therefore, in that kinematical regime those densities cannot be determined well, and large discrepancies between different analyses are expected. For the gluon fragmentation, the main source of information at $z \gtrsim 0.5$ stems from PHENIX data at mid and STAR and BRAHMS data at forward rapidity. These are included only in the analysis presented here. BRAHMS and STAR data access the largest $z$ values of all data sets in our global analysis. As was observed in Figs. 5 - 7. the KRE sets underestimates all experimental data (for $\mu_{f}=\mu_{r}=p_{T}$ ), while the AKK set agrees with the PHENIX data but tends to underestimate STAR. This explains the differences in Figs. 17 and 18 between our gluon fragmentation function and those of KRE and AKK. RHIC data tend to favor a significantly larger $D_{g}^{\pi^{+}}$ at large $z$.

Finally, the comparison between the different distributions and ratios in Figs. 17 and 18 also highlights the importance of the $Q^{2}$ evolution. Even though the distributions show a much stronger rise at small $z$ when the scale is increased [49], one also finds a much better agreement between the different sets at $Q^{2}=M_{Z}^{2}$, where most of the very precise SIA data are obtained. In other words, the evolution downwards from $M_{Z}$, were the distributions agree best, to scales relevant for RHIC and SIDIS data, exacerbate the differences between them.

Figures 19 and 20 provide the same information and comparisons as in the previous ones but for positively charged kaons. As expected, the dominant fragmentation in the light quark sector corresponds to the strange distribution. While heavy quark densities are as large as those for pions, the gluon fragmentation turns out to be much less sizable, even though it is still larger than those from KRE and AKK at large $z$ in order to fit the protonproton data from STAR and, in particular, at forward rapidities from BRAHMS. The comparison in the light quark sector shows many similarities with the pion case, but here the discrepancies are more noticeable with the KRE set instead. The global fit requires a smaller contribution from $u$ quark fragmentation, mostly from SIDIS data (distributions like KRE overestimate SIDIS data, see Fig. 13) resulting in an increase in the strange sector as the singlet $D_{\Sigma}^{K^{+}}$is again constrained by SIA data.

\section{F. Uncertainties}

In order to give a clear and comprehensive picture of the typical uncertainties characteristic of the fragmentation functions obtained in the global fits, in the present Section we apply the Lagrange multiplier technique introduced in Sec. IIID.

Rather than focusing on the uncertainties of the parameters in Eq. (15) determining the fragmentation functions at the initial scale and choosing a particular increment $\Delta \chi^{2}$ to judge the quality of the fit, we find it much more enlightening to analyze the range of variation of other relevant features of the fragmentation functions, with a more apparent physical meaning, and take these as the characteristic uncertainties of the fit. Notice that the range of variation of the fitted parameters are strongly correlated; the impact of any of them on the behavior of the distributions, or on a given observable, is determined also by the values taken by the whole set of parameters through the evolution equations. Of course, in order to get a precise estimate of the uncertainty in a given observable computed with the set, the range of variation of that particular observable as a function of $\Delta \chi^{2}$ has to be evaluated. As explained in Sec. IIID the result takes into account the complex correlations between the parameters, implies no assumptions on the profile of $\chi^{2}$, and allows to consider different $\Delta \chi^{2}$.

In Figures 21 and 22 we show, as an example, the range of variation of the truncated second moments of the fragmentation functions

$$
\eta_{i}^{H}\left(x_{p}, Q^{2}\right) \equiv \int_{x_{p}}^{1} z D_{i}^{H}\left(z, Q^{2}\right) d z,
$$

for $x_{p}=0.2$ and $Q=5 \mathrm{GeV}$, around the values obtained for them in the best fit to data, $\eta_{i 0}^{H}$, against the corresponding increase in $\chi^{2}$. In the lowest order, the second moments represent the energy fraction of the parent parton of flavor $i$ taken by the hadron $H$. The truncated moments discard the low- $z$ contributions, which are not constrained by data and therefore only determined by low- $z$ extrapolation.

As it can be seen in the upper left panel of Fig. 21, the truncated moment $\eta_{u+\bar{u}}^{\pi^{+}}$, associated with $D_{u+\bar{u}}^{\pi^{+}}$, is the constrained best, with a range of variation of less than $3 \%$ around the value computed with the best fit, assuming a very conservative increase in $\chi^{2}$ by 15 units, i.e., $\Delta \chi^{2}=15$. This comparatively stringent restriction comes from the fact that all the observables accounted for in the fit have a strong dependence on the corresponding $D_{u+\bar{u}}^{\pi^{+}}$fragmentation function. Moving to the next panel, we find that for the unfavored $D \frac{\pi^{+}}{u}$ fragmentation function, the truncated moment is less, but still well constrained within a $5 \%$ range for a similar $\Delta \chi^{2}$. While this distribution cannot be determined by SIA data without flavor symmetry assumptions, it is constrained by SIDIS and proton-proton collisions involving low $x$ contributions, where the $\bar{u}(x)$ PDF is large, or at high $x$ 


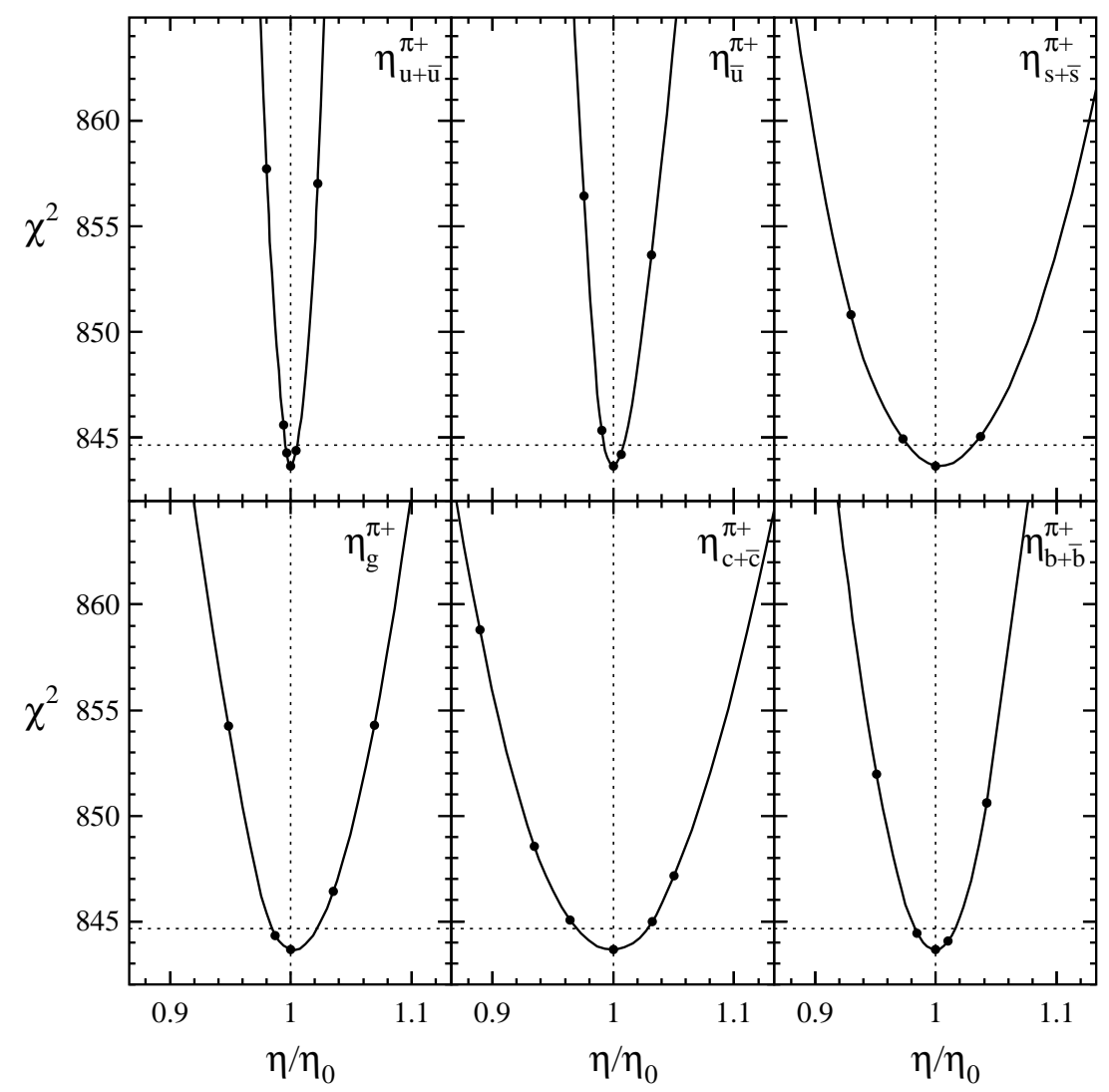

FIG. 21: Profiles of $\chi^{2}$ for the NLO pion fragmentation fit as a function of the truncated second moments $\eta_{i}^{\pi^{+}}\left(x_{p}=0.2, Q=\right.$ $5 \mathrm{GeV}$ ) for different flavors. The moments are normalized to the value $\eta_{i 0}^{\pi^{+}}$they take in the best fit to data.

through its alter ego $D_{u}^{\pi^{-}}$.

The fragmentation functions for strange quarks have much larger uncertainties, and their moment can vary by more that $10 \%$, as shown in the upper right panel of Fig. 21. In our fit, not only $D \frac{\pi^{+}}{u} \neq D \frac{\pi}{s}^{+}$but their respective uncertainties are found to be quite different as well. Notice that these uncertainties in the unfavored distributions cannot justify the differences with KRE and AKK sets found in the previous Section.

The moment for the gluon fragmentation function, in the lower left panel of Fig. 211 is restricted to vary by less than $10 \%$, with the constraint mainly stemming from the evolution from the initial scale to the scales relevant for each measurement, rather than from a direct contribution to a particular cross section. Such kind of contributions are certainly present in RHIC data, but only in narrow intervals of $z$, so they cannot fix the truncated moment for $x_{p}=0.2$ by themselves. For heavy quarks, flavor tagged data dominate the fit, and the more precise b-tagged data lead to better constrained moments for $D_{b+\bar{b}}^{\pi^{+}}$than for $D_{c+\bar{c}}^{\pi^{+}}$.

The uncertainties in kaon fragmentation functions, Fig. 22, are typically twice as large as those for pions, with $10 \%$ variations for the total fragmentation functions containing a favored fragmentation function and rather poorly constrained unfavored fragmentation functions $D_{\bar{u}}^{K^{+}}=D_{\bar{d}}^{K^{+}}=D_{d}^{K^{+}}$. The profiles show much more significant deviations from the parabolic behavior than in the case of pions, and the upper bound for the moment of the gluon distribution is much less defined.

Next we further illuminate the role of the different data sets in setting the constraints on the fragmentation functions. In Figs. 23 and 24 we show the partial contributions $\Delta \chi_{i}^{2}$ of a data set $i$ to the increase of $\chi^{2}$ from its minimum value defined by the best fit against the variation of some selected truncated moments.

In the upper left panel of Fig. 23 it can be noticed that both SIA (thick solid line) and SIDIS (thick dashed dotted line) data define their own minima for the $u+\bar{u}$ moment, slightly to the right and to the left of the best fit value, respectively, but within the above mentioned conservative uncertainty range of $\Delta \chi^{2}=15$. The other data sets fail to develop well defined minima (at least within the shown range), and the final result is a compromise between all of them.

Notice that the failure to define a minimum by a given 


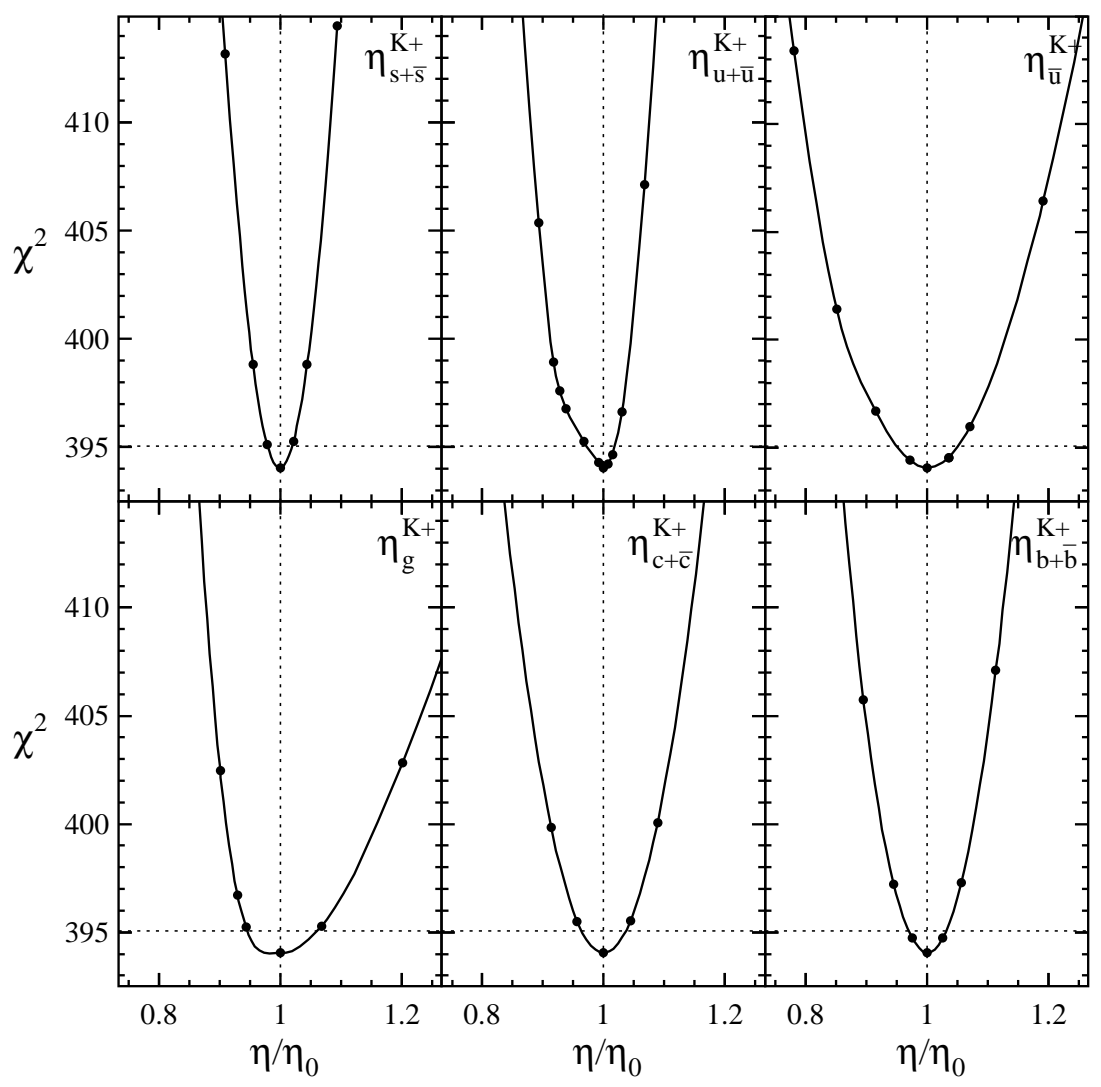

FIG. 22: The same as in Fig. 21, but now for kaon fragmentation at NLO.

subset of data may not necessarily imply a weak dependence of the data on the particular flavor that fragments. It may happen because the data cover a limited range in $z$, while the observable we chose picks up contributions over a much wider range in $z$. The seemingly contradictory "preferences" of two data sets can follow from sensitivity to complementary regions in $z$.

The truncated moment for the gluon-to-pion fragmentation function, shown in the upper right panel of Fig.23, happens to be well constrained again by SIA and SIDIS data, with their respective minima very close to the best fit result. For $\eta_{s+\bar{s}}^{\pi^{+}}$, in the lower right panel, neither set shows a minimum and the final result is a compromise. Notice that, here, the most significant contributions are those coming from SIA and the OPAL tagging probabilities.

Partial contributions $\Delta \chi_{i}^{2}$ to the truncated moments for kaon fragmentation functions, Fig. 24, have less definite preferences, leading to much larger uncertainties. Starting with the moment for $s+\bar{s}$ in the upper left panel, we find that SIDIS data now fail to define a minimum. This is mainly due to the limited strangeness content in the proton. However, SIDIS contributes with OPAL tagged data to balance the preference coming from
SIA data, which is slightly larger than the best fit value. Gluon-to-kaon fragmentation, shown in the upper right panel, is mostly constrained by STAR and SIDIS data. The $u+\bar{u}$ moment, receiving contributions from the suppressed $D_{u}^{K^{+}}$and the doubly suppressed $D_{\bar{u}}^{K^{+}}$, is neither well constrained by SIA nor by SIDIS data, however, OPAL and STAR data help to improve the situation.

Upon the completion of our analysis, a new determination of fragmentation functions, again based only on SIA data but with a careful assessment of uncertainties, was published [10]. There, it is shown that the large differences found between the most widely used sets (KRE, $\mathrm{KKP}$, and AKK) are related to the large $z$ behavior of the fragmentation functions, where the uncertainties are found to be most significant. Indeed, we can see that this is the case for sets based only in SIA data, but the situation is considerably improved in a global fit. In the left panel of Fig. 25] we show, as an example, the partial contributions $\Delta \chi_{i}^{2}$ from different sets of data to the truncated moment for $D_{u+\bar{u}}^{\pi^{+}}$, but now taking only large$z$ contributions, $x_{p}=0.5$ in Eq. (29). Here it can be seen that SIA data, as expected, fail to define a minimum, but the complementary information coming from the other sets, dominated by PHENIX and SIDIS, de- 


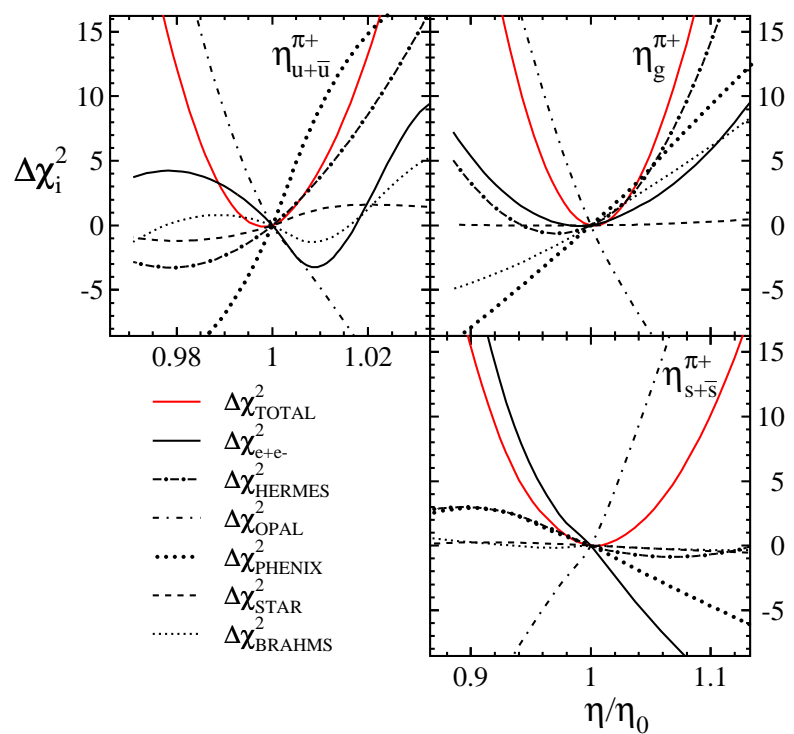

FIG. 23: Partial contributions $\Delta \chi_{i}^{2}$ of the individual data sets to the NLO global analysis of pion fragmentation functions against the variation in selected truncated moments.

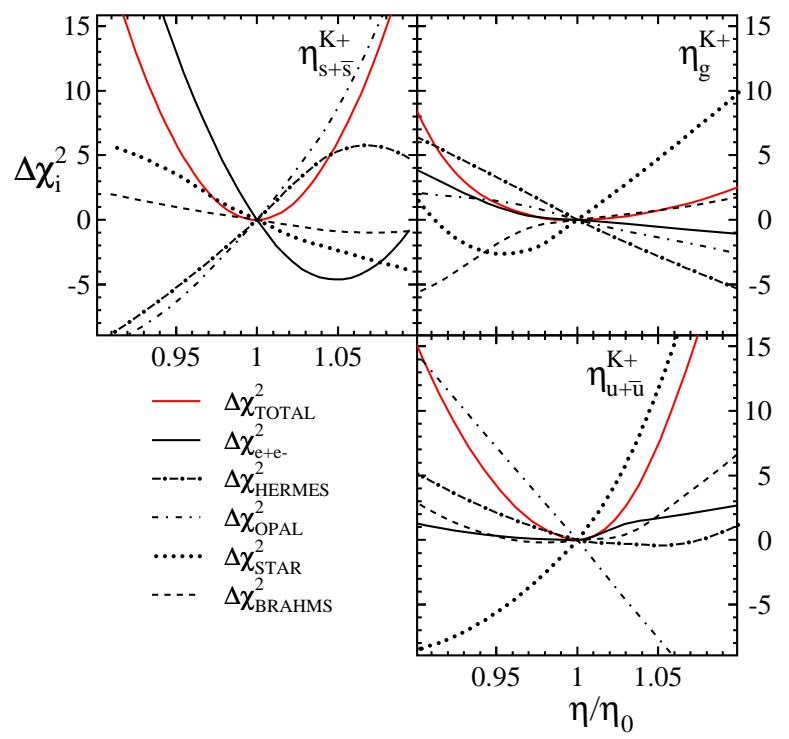

FIG. 24: The same as in Fig. 23. but for NLO kaon fits.

fine a clear minimum, with a well a constrained range of variation, again showing the power and importance of a global analysis.

Another interesting difference with the analysis of Ref. [10] is that while they accomplish a good fit to SIA data under the assumption $N^{\prime}=1$ in Eq. (18), our global fit prefers $N^{\prime} \simeq 0.83$. In order to understand this difference, the right panel of Fig. 25 shows the partial contribution to $\chi^{2}$ for different values of $N^{\prime}$ normalized to $N^{\prime}$ for our best fit. Although the fit clearly prefers $N^{\prime} \simeq 0.83$, the uncertainty is large, and for a very conservative $\Delta \chi^{2}$ may be taken as marginally consistent with $N^{\prime}=1$. The

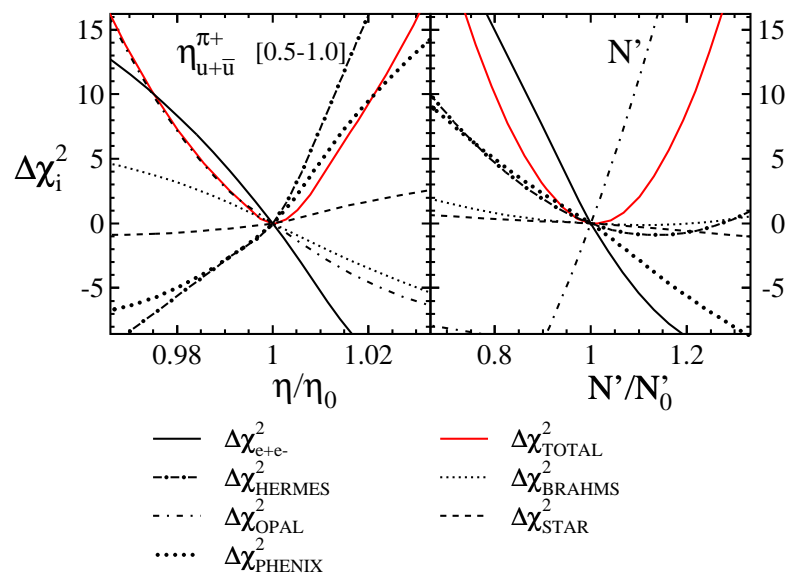

FIG. 25: The same as in Fig. 223 but for $\eta_{u+\bar{u}}^{\pi^{+}}\left(x_{p}=0.5, Q=\right.$ $5 \mathrm{GeV}$ ) (left panel) and $N^{\prime}$ (right panel).

preference for $N^{\prime}$ values smaller than one in our global fit is driven by the OPAL tagging probabilities, not included in [10].

Finally, we note that projected measurements of hadron production in SIA by the BELLE and/or the BaBar experiments at low c.m.s. energies would open up the possibility for studies of scaling violations with unprecedented precision. Such data should help to further constrain the fragmentation functions and significantly reduce the present uncertainties.

\section{CONCLUSIONS}

We have demonstrated the feasibility of a NLO combined QCD analysis of single inclusive hadron production data for pions and kaons, coming from electronpositron annihilation, deep-inelastic lepton-nucleon scattering, and proton-proton collisions, collected over a wide kinematic range.

At variance with previous fits based only on electronpositron annihilation data, the present analysis includes complementary information from other experiments that reduce significantly the uncertainties of the resulting fragmentation functions.

In the case of pion fragmentation functions, we find that the new SIDIS data provided by the HERMES experiment, effectively constrain the separation between favored and unfavored distributions, a separation that was either not implemented in previous sets or it was based on certain assumptions. The most recent RHIC results provide stringent constraints on the gluon fragmentation function and, in general, on the large $z$ behavior of the other distributions. For kaons, the new data modify significantly the up-to-now standard picture provided by previous analyses. Specifically, SIDIS data rule out the flavor separation scheme hitherto implemented, while RHIC data lead to a new gluon fragmentation function, and thus scale dependences, significantly different over 
the whole range of $z$.

The implementation of the $\chi^{2}$ minimization in our global analysis is numerically fast and efficient and can be straightforwardly expanded to any future set of hadron production data. With the help of the Mellin moment technique, the entire analysis was consistently performed at NLO accuracy without resorting to often used approximations for NLO hard scattering cross sections.

The success of the global analysis performed here, for the first time including observables other than single inclusive annihilation, stands for an explicit check of factorization, universality, and the perturbative QCD framework for the description of the corresponding processes, providing at the same time much more precise constraints on the fragmentation functions. Proton-proton collision data and that coming from SIDIS offer a crucial piece of information that cannot be disregarded and will be increasingly accessible in the near future.

\section{Acknowledgments}

We warmly acknowledge Elke Aschenauer and Achim Hillenbrand for help with the HERMES data, Werner Vogelsang for helpful discussions, and Carlos García Canal for comments and suggestions. This work was partially supported by CONICET, ANPCyT and UBACyT.
[1] See, e.g., J.C. Collins, D.E. Soper, and G. Sterman, "Perturbative QCD", A.H. Mueller (ed.), Adv. Ser. Direct. High Energy Phys. 5, 1 (1988) and references therein.

[2] J.C. Collins and D.E. Soper, Nucl. Phys. B193, 381 (1981); B213, 545(E) (1983); B194, 445 (1992).

[3] J.M. Campbell, J.W. Huston, and W.J. Stirling, Rept. Prog. Phys. 70, 89 (2007).

[4] See, e.g., A. Vogt, S. Moch, and J. Vermaseren, Nucl. Phys. Proc. Suppl. 160, 44 (2006),

[5] R.S. Thorne, A.D. Martin, and W.J. Stirling, hep-ph/0606244.

[6] J. Pumplin, A. Belyaev, J. Huston, D. Stump, and W.K. Tung, JHEP 0602, 032 (2006).

[7] S. Kretzer, Phys. Rev. D62, 054001 (2000).

[8] B.A. Kniehl, G. Kramer, and B. Pötter, Nucl. Phys. B582, 514 (2000).

[9] S. Albino, B.A. Kniehl, and G. Kramer, Nucl. Phys. B725, 181 (2005); B734,50 (2006).

[10] M. Hirai, S. Kumano, T.-H. Nagai, and K. Sudoh, hep-ph/0702250

[11] ALEPH Collaboration, D. Buskulic et al., Z. Phys. C66, 355 (1995).

[12] DELPHI Collaboration, P. Abreu et al., Eur. Phys. J. C5, 585 (1998).

[13] OPAL Collaboration, R. Akers et al., Zeit. Phys. C63, 181 (1994).

[14] OPAL Collaboration, G. Abbiendi et al., Eur. Phys. J. C16, 407 (2000).

[15] TP Collaboration, H. Aihara et al., Phys. Rev. Lett. 61, 1263 (1998); Phys. Lett. B184, 299 (1987); X.-Q. Lu, Ph.D. thesis, John Hopkins University, UMI-87-07273, 1986.

[16] SLD Collaboration, K. Abe et al., Phys. Rev. D59, 052001 (1999).

[17] TASSO Collaboration, W. Braunschweig et al., Zeit. Phys. C42, 189 (1989).

[18] A. Hillenbrand (HERMES Collaboration), "Measurement and Simulation of the Fragmentation Process at HERMES", Ph.D. thesis, Erlangen Univ., Germany, September 2005; private communications.

[19] PHENIX Collaboration, S.S. Adler et al., Phys. Rev. Lett. 91, 241803 (2003); K. Barish (PHENIX Collaboration), "The PHENIX Spin Program: Recent Results and Future Prospects", talk presented at SPIN 2006, Kyoto,
Japan.

[20] STAR Collaboration, J. Adams et al., Phys. Rev. Lett. 97, 152302 (2006).

[21] BRAHMS Collaboration, I. Arsene et al., hep-ex/0701041

[22] STAR Collaboration, B.I. Abelev et al.,. nucl-ex/0607033

[23] J. Pumplin, D.R. Stump, and W.-K. Tung, Phys. Rev. D65, 014011 (2002); D. Stump et al., Phys. Rev. D65, 014012 (2002).

[24] D. de Florian, M. Stratmann, and W. Vogelsang, Phys. Rev. D57, 5811 (1998).

[25] B. Jäger, A. Schäfer, M. Stratmann, and W. Vogelsang, Phys. Rev. D67, 054005 (2003).

[26] P.J. Rijken and W.L. van Neerven, Nucl. Phys. B487, 233 (1997); Phys. Lett. B386, 422 (1996).

[27] G. Curci, W. Furmanski, and R. Petronzio, Nucl. Phys. B175, 27 (1980); W. Furmanski and R. Petronzio, Phys. Lett. 97B, 437 (1980); L. Beaulieu, E.G. Floratos, and C. Kounnas, Nucl. Phys. B166, 321 (1980).

[28] M. Stratmann and W. Vogelsang, Nucl. Phys. B496, 41 (1997).

[29] G. Altarelli, R.K. Ellis, G. Martinelli, and S.Y. Pi, Nucl. Phys. B160, 301 (1979); P. Nason and B. Webber, Nucl. Phys. B421, 473 (1994); B480, 755(E) (1996).

[30] W. Furmanski and R. Petronzio, Z. Phys. C11, 293 (1982).

[31] D. Graudenz, Nucl. Phys. B432, 351 (1994).

[32] L. Trentadue and G. Veneziano, Phys. Lett. B323, 201 (1993); D. de Florian, C. García Canal, and R. Sassot, Nucl. Phys. B470, 195 (1996); D. de Florian and R. Sassot, Nucl. Phys. B488, 367 (1997).

[33] F. Aversa, P. Chiappetta, M. Greco, and J.-Ph. Guillet, Nucl. Phys. B327, 105 (1989); D. de Florian, Phys. Rev. D67, 054004 (2003).

[34] E. Christova, S. Kretzer, and E. Leader, Eur. Phys. J. C22, 269 (2001).

[35] M. Cacciari, P. Nason, and C. Oleari, JHEP 0510, 034 (2005).

[36] A.D. Martin, R.G. Roberts, W.J. Stirling, and R.S. Thorne, Eur. Phys. J. C28, 455 (2003).

[37] A.D. Martin, R.G. Roberts, W.J. Stirling, and R.S. Thorne, Phys. Lett. B531, 216 (2002).

[38] For a compilation of SIA data, see, G.D. Lafferty, P.I. 
Reeves, and M.R. Whalley, J. Phys. G21, A1 (1995).

[39] M. Glück, E. Reya, and A. Vogt, Z. Phys. C48, 471 (1990); Phys. Rev. D45, 3986 (1992).

[40] M. Glück, E. Reya, and A. Vogt, Phys. Rev. D48, 116 (1993); D51, 1427(E) (1995).

[41] M. Stratmann and W. Vogelsang, Phys. Rev. D64, 114007 (2001).

[42] C. Berger, D. Graudenz, M. Hampel, and A. Vogt, Z. Phys. C70, 77 (1996); D.A. Kosower, Nucl. Phys. B520 (1998) 263.

[43] M. Botje, Eur. Phys. J. C14 (2000) 285.

[44] D. de Florian, G.A. Navarro, and R. Sassot, Phys. Rev. D71, 094018 (2005); G.A. Navarro and R. Sassot, Phys. Rev. D74, 011502 (2006); X. Jiang, G.A. Navarro, and
R. Sassot, Eur. Phys. J. C47, 81 (2006).

[45] V. Guzey, M. Strikman, and W. Vogelsang, Phys. Lett. B603, 173 (2004).

[46] H1 Collaboration, A. Aktas et al., Eur. Phys. J. C36, 441 (2004).

[47] A. Daleo, D. de Florian, and R. Sassot, Phys. Rev. D71, 034013 (2005).

[48] A Fortran package containing our LO and NLO sets of pion and kaon fragmentation functions can be obtained upon request from the authors.

[49] Recall that, as discussed in Sec. IA at much smaller values of $z$ the NLO splitting kernels produce negative distributions and hadron mass effects become important. 